Homology, Homotopy and Applications, vol.3, No.3, 2001, pp.55-85

\title{
TENSOR PRODUCTS OF SPECTRA AND LOCALIZATIONS
}

\author{
FRIEDRICH W. BAUER
}

(communicated by Walter Tholen)

\begin{abstract}
1) The tensor product of two spectra, different from the $\wedge-$ product, is introduced in such a way that a Künneth theorem holds. 2) Localizations of spectra are treated by using the more algebraic category of chain functors (instead of the category of CW-spectra). 3) The localization of a given chain functor $\mathbf{K}_{*}$ can up to chain homotopy be expressed by tensoring $\mathbf{K}_{*}$ with the localization of a fixed chain functor $\mathbb{Z}_{*}$.
\end{abstract}

\section{Introduction}

Concerning details about chain functors we refer to [5] $\S 4$, resp. $\S 5$ for the motivation.

A chain functor $\boldsymbol{K}_{*}=\left\{K_{*}, K_{*}^{\prime}, i^{\prime}, l, \varphi, \kappa\right\}$ consists of a functor

$$
K_{*}: \mathfrak{K} \longrightarrow \mathbf{c h} \text { (= category of chain complexes), }
$$

$\mathfrak{K}$ a suitable category of pairs of topological spaces or of spectra, a subfunctor $l: K_{*}^{\prime} \subset K_{*}$, a natural inclusion $i^{\prime}: K_{*}(A) \subset K_{*}^{\prime}(X, A)$ together with some other structural (non-necessary natural) mappings $\varphi: K_{*}^{\prime}(X, A) \longrightarrow K_{*}(X), \kappa:$ $K_{*}(X) \longrightarrow K_{*}^{\prime}(X, A)$, satisfying certain properties. Every homology theory $h_{*}()=$ $\left\{h_{n}(), \partial, n \in \mathbb{Z}\right\}$ admits a chain functor such that the derived homology homology $H_{*}\left(K_{*}(X, A)\right)$ is naturally isomorphic to $h_{*}(X, A)$. In addition the boundary $\partial$ : $h_{*}(X, A) \longrightarrow h_{n-1}(A)$ is determined by the chain functor $\boldsymbol{K}$. This last property distinguishes chain functors from simple functors (1) admitting natural sequences

$$
0 \longrightarrow K_{*}(A) \stackrel{i_{*}}{\longrightarrow} K_{*}(X) \stackrel{j_{*}}{\longrightarrow} K_{*}(X, A) \longrightarrow 0
$$

giving rise to a boundary operator

$$
\partial: H_{*}\left(K_{*}(X, A)\right) \longrightarrow H_{n-1}\left(K_{*}(A)\right)
$$

(called chain theories, which are only available for ordinary, but not necessarily for generalized homology theories).

In the present paper we are dealing with the following three objectives:

1) Definition of tensor products of spectra in such a way that, unlike for $\wedge$ - products, a Künneth-theorem holds $(\S 1,2)$.

Received February 24, 2000, revised November 23, 2000; published on February 3, 2001.

2000 Mathematics Subject Classification: 55P60, 55P42, 18G15, 18 G35.

Key words and phrases: tensor products; homology theories associated with a chain functor; localizations of chain functors; regular and irregular-chain-functors.

(c) 2001, Friedrich W. Bauer. Permission to copy for private use granted. 
2) Establishing localizations of chain functors and verification of the Bousfield exact sequence of a localization but now for chain functors instead of spectra (§5).

Finally:

3) Expressing localizations of chain functors by tensoring them with the localization of a specific chain functor (cf. $\S 3$ resp. $\S 6$ ). Therefore localizations can, at least up to a chain homotopy, be described, similar to the algebraic case of group-localization, by means of a tensor product (cf. [11] appendix).

Although our main objective lies in the localization of CW-spectra (i.e. of elements of the Boardman category), we deal mainly with chain functors, recalling that there is a close relationship between $\mathrm{CW}$-spectra $\mathbf{A}$, the associated homology theory $\mathbf{A}_{*}$ and the chain functors $\mathbf{C}_{*}$ whose related homology theory $H_{*}\left(\mathbf{C}_{*}\right)$ is isomorphic to $\mathbf{A}_{*}$ (cf. $\S 4$ in ). More precisely: Every generalized homology theory $h_{*}$ (even on $\mathbf{T o p}^{2}$ or a suitable subcategory) originates from a chain functor $\mathbf{C}_{*}$ such that $h_{*} \approx H_{*}\left(\mathbf{C}_{*}\right)$ (cf. theorem 8.1. in [2]) while on the other hand, each chain functor $\mathbf{C}_{*}$ with compact carrier on $\mathbf{C W}^{2}=\mathfrak{P}^{2}$, the category of $\mathrm{CW}$-pairs defines a $\mathrm{CW}$ spectrum $\left|\mathbf{C}_{*}\right|$ such that $\left|\mathbf{C}_{*}\right|_{*} \approx H_{*}\left(\mathbf{C}_{*}\right)$ (theorem 1.1. in [3]).

Together with the first result this leads to E. H. Brown's representation theorem for homology theories.

In $\S 1$ we introduce the tensor product of chain functors $\mathbf{K}_{*} \otimes \mathbf{L}_{*}$ which is defined in analogy to the classical $\otimes$-product of chain complexes (cf. [8]).

Due to this fact there is a Künneth theorem (theorem 1.7.) for tensor products of chain functors.

The tensor-product of spectra $\mathbf{A}, \mathbf{B}$ is defined in $\S 2$ by assigning to $\mathbf{A}_{*}, \mathbf{B}_{*}$, the associated homology theories, the chain functors ${ }^{A} \mathbf{C}_{*},{ }^{B} \mathbf{C}_{*}$ and setting

$$
\mathbf{A} \otimes \mathbf{B}=\left|{ }^{A} \mathbf{C}_{*} \otimes{ }^{B} \mathbf{C}_{*}\right| .
$$

The independence of $\mathbf{A} \otimes \mathbf{B}$ of the choices of ${ }^{A} \mathbf{C}_{*},{ }^{B} \mathbf{C}_{*}$ can be confirmed by using the main result of [5], where among other things the concept of a chain functor, a mapping between chain functors as well as some other relevant details are recorded. In particular, we call chain functors, whose associated homology satisfies an excision axiom (hence becoming in this way a generalized homology theory) regular. Moreover there is need for structures which do not enjoy all properties of a chain functor (in particular not of a regular one). We call, by an abuse of notation, these structures irregular-chain-functors (cf. [5] definition 4.1.2). Unlike in [5] we do not insist, that a regular chain functor has automatically compact carriers.

In $\S 3$ we present an example of such an irregular-chain-functor $\mathbb{Z}_{*}$ (which is not associated with any spectrum, because the realization theorem does not apply) having the property that for any chain functor $\mathbf{K}_{*}$ one obtains an isomorphism of irregular chain functors

$$
\mathbf{K}_{*} \otimes \mathbb{Z}_{*} \approx \mathbf{K}_{*}
$$

(theorem 3.1.). In $\S 5$ we describe the process of $\mathfrak{L}$-localization with respect to a subcategory $\mathfrak{L} \subset \mathfrak{K}^{2}$ (= the category on which the chain functors are defined). Theorem 5.5. asserts the existence of a Bousfield exact sequence for the localizations of chain functors:

$$
\mathfrak{L} \mathbf{A}_{*} \longrightarrow \mathbf{A}_{*} \longrightarrow \mathbf{A}_{\mathfrak{L} *}
$$


with $\mathfrak{L}$-acyclic ${ }_{\mathfrak{L}} \mathbf{A}_{*}$ and $\mathfrak{L}$ - local $\mathbf{A}_{\mathfrak{L} *}$. (cf. definition 4.4. resp. 5.1.).

We may regard as a special case the category $\mathfrak{L}$ consisting of one single object $E \in \mathfrak{K}$ (with the identity as single morphism) (corollary 5.6.).

If $\mathfrak{K}^{2}$ is a subcategory of Top ${ }^{2}$ and $\mathbf{E} \in \mathfrak{B}$ (=Boardman category) a spectrum, then we can look upon $\mathbf{E}$ as a specific subcategory of $\mathfrak{K}$ and define $\mathbf{A}_{E *}$ by using this category (corollary 5.8.).

In $\S 6$ we prove the main theorem (theorem 6.5 .) asserting that the $\mathfrak{L}$-localization sequence (4) in §5) can be achieved, up to a chain homotopy equivalence, by tensoring $\mathbf{A}_{*}$ with the specific $\mathfrak{L}$-localization of $\mathbb{Z}_{*}$.

This constitutes the topological analogue of a well-known algebraic result about localizations of a group at a set of primes.

Recently the authors of [9] developed another algebraic approach to stable homotopy theory together with an appropriate concept of a $\wedge$ - product.

The proofs in $\S 5, \S 6$ are preceeded by some general results in $\S 4$.

All notations and definitions concerning chain functors are recorded in [5] $\S 4$ to which we freely refer without further mentioning.

The results of the present paper are reported in the expository article [4].

\section{Tensor products of chain functors}

The concept of a chain functor and the associated homology is recorded in $\S 4$.

Let $\mathbf{K}_{*}, \mathbf{L}_{*}$ be regular or irregular-chain-functors as defined in [5] $\S 4$, definition 4.1., then we define their tensor product $(\mathbf{K} \otimes \mathbf{L})_{*}$ :

Suppose $(X, A) \in \mathfrak{K}^{2}$, then we set

$$
\begin{array}{r}
(K \otimes L)_{n}(X, A)=\bigoplus_{p+q=n} K_{p}(X, A) \otimes L_{q}(X, A) \oplus \\
\oplus \bigoplus_{p+q=n} K_{p}(X, A) \otimes L_{q}(X) \oplus \bigoplus_{p+q=n} K_{p}(X) \otimes L_{q}(X, A)
\end{array}
$$

where the boundary

$$
d_{n}:(K \otimes L)_{n}(X, A) \longrightarrow(K \otimes L)_{n-1}(X, A)
$$

is defined as usual for tensor products of chain complexes.

These direct sums are to be understood as amalgamated sums whenever inclusions like $j: X \subset(X, A)$ which induce inclusions (e.g. for regular chain complexes) are involved: So, for example,

$$
x \otimes y \in K_{p}(X, A) \otimes L_{q}(X)
$$

is identified with

$$
x \otimes j_{\#}(y) \in K_{p}(X, A) \otimes L_{q}(X, A)
$$

As a result for regular chain functors this reduces to:

$$
(K \otimes L)_{n}(X, A)=\bigoplus_{p+q=n} K_{p}(X, A) \otimes L_{q}(X, A)
$$


which is the ordinary tensor product of chain complexes (cf. [8]).

We define:

$$
\begin{array}{r}
(K \otimes L)_{n}^{\prime}(X, A)=\bigoplus_{p+q=n} K_{p}^{\prime}(X, A) \otimes L_{q}^{\prime}(X, A) \oplus \\
\oplus \bigoplus_{p+q=n} K_{p}^{\prime}(X, A) \otimes L_{q}(A, A) \oplus \bigoplus_{p+q=n} K_{p}(A, A) \otimes L_{q}^{\prime}(X, A) .
\end{array}
$$

Since $K_{*}(\cdot)$ and $L_{*}(\cdot)$ are free, we have for regular chain functors an inclusion

$$
l:(K \otimes L)_{*}^{\prime}(X, A) \subset(K \otimes L)_{*}(X, A)
$$

For irregular-chain-functors there is still a natural $l$ available, which is not monic. The mappings $i^{\prime K}: K_{*}(A) \longrightarrow K^{\prime}{ }_{*}(X, A)$ resp. for $L$ induce a chain mapping $i^{\prime}:(K \otimes L)_{*}(A) \longrightarrow(K \otimes L)_{*}^{\prime}(X, A)$, which for regular $\mathbf{K}_{*}, \mathbf{L}_{*}$ is an inclusion. The chain mapping $\varphi:(K \otimes L)_{*}^{\prime}(X, A) \longrightarrow(K \otimes L)_{*}(X)$ is $\varphi^{K} \otimes \varphi^{L}$ on the first summand of $(2)\left(\varphi^{K}, \varphi^{L}\right.$ denoting the corresponding maps for $\left.\mathbf{K}_{*}, \mathbf{L}_{*}\right)$ and it is trivial on the remaining two summands $\bigoplus_{p+q=n} \cdots$.

$$
\kappa:(K \otimes L)_{*}(X) \longrightarrow(K \otimes L)_{*}^{\prime}(X, A)
$$

maps the first summand of (1) by $\kappa^{K} \otimes \kappa^{L}$.

We are now going to verify the properties of a chain functor as they were recorded in $[5], \S 4$ :

Since we have chain homotopies $\varphi^{K} \kappa^{K} \simeq 1$ resp. for $L$, the existence of a chain homotopy (as required in D1) [5], §4)

$$
\varphi \kappa \simeq 1
$$

follows.

We need

1.1. Lemma: Let $\mathbf{C}_{*}$ be any chain functor, $d c=k z$, in $C_{*}(X, A), k \in \mathbb{Z}$, then there exist: $z^{\prime}, c^{\prime} \in C_{*}^{\prime}(X, A), a_{i} \in C_{*}(A, A), i=1,2, a_{3} \in C_{*}(A)$ such that

$$
\left\{\begin{array}{l}
l z^{\prime}+q_{\#} a_{1} \sim z, \quad d z^{\prime} \in i m i^{\prime} \\
l c^{\prime}+q_{\#} a_{2}=c+d w, \quad w \in C_{*}(X, A) \\
k z^{\prime}+i^{\prime} a_{3}=d c^{\prime} .
\end{array}\right.
$$

Proof: There exists $z \sim l z^{\prime}+q_{\#} a_{1}$ so that without loss of generality we are allowed to assume that $z=l z^{\prime}+q_{\#} a_{1}$. Because of [5] $\S 4$ there exist non-natural chain homotopies $j_{\#} \varphi \simeq l, \varphi \kappa \simeq 1$, hence a chain homotopy (writing simply $j$ for $\left.j_{\#}\right)$

$$
l \kappa \varphi \simeq j \varphi \kappa \varphi \simeq j \varphi \simeq l .
$$

More precisely there are due to [5], $\S 4$ chains $D\left(z^{\prime}\right), \quad D\left(d z^{\prime}\right)$ such that

$$
l \kappa \varphi z^{\prime}-l z^{\prime}=d D\left(z^{\prime}\right)+D\left(d z^{\prime}\right) .
$$

Since $d z^{\prime} \in i m i^{\prime}$, we can, because of [5] $\S 4$, D1"), assume that $D\left(d z^{\prime}\right)=0$ so that

$$
z=l z^{\prime}+q_{\#} a_{1} \sim l \kappa \varphi z^{\prime}+q_{\#} a_{1} .
$$


By substituting $z$ we assume without loss of generality that

$$
z=l \kappa \varphi z^{\prime}+q_{\#} a_{1} .
$$

Since $k z=d c$, D3) (in [5] §4) implies the existence of $\bar{a} \in C_{*}(A)$ such that

$$
\begin{gathered}
d k \kappa \varphi z^{\prime}=-i^{\prime} d \bar{a}, \\
k z=k l \kappa \varphi z^{\prime}+l i^{\prime} \bar{a}+\left(q_{\#} a_{1}-l i^{\prime} \bar{a}\right) \\
=k l \kappa \varphi z^{\prime}+l i^{\prime} \bar{a}+d v
\end{gathered}
$$

taking into account that $q_{\#} k a_{1}-l i^{\prime} \bar{a} \in C_{*}(A, A)$ is a cycle, hence bounding (since $C_{*}(A, A)$ is acyclic).

So

$$
k z=l\left(k \kappa \varphi z^{\prime}+i^{\prime} \bar{a}\right)+d v, \quad v \in C_{*}(A, A) .
$$

However, since $k z$, hence $l\left(k \kappa \varphi z^{\prime}+i^{\prime} \bar{a}\right)$ is bounding, D2) in [5] $\S 4$ ensures the existence of a $c_{1}^{\prime} \in C_{*+1}^{\prime}(X, A), \quad b \in C_{*}(A)$ such that

$$
k \kappa \varphi \kappa \varphi z^{\prime}+\kappa \varphi i^{\prime} \bar{a}=d c_{1}^{\prime}+i^{\prime}(b) .
$$

Due to [5] $§ 4$ D1') we can assume

$$
\kappa \varphi i^{\prime}=\kappa i=i^{\prime}
$$

hence

$$
k \kappa \varphi \kappa \varphi z^{\prime}+\kappa \varphi i^{\prime} \bar{a} \sim k \kappa \varphi z^{\prime}+i^{\prime} \bar{a} .
$$

As a result we find a $c_{2}^{\prime} \in C_{*}^{\prime}(X, A)$ satisfying

$$
\begin{gathered}
d c_{2}^{\prime}=k \kappa \varphi z^{\prime}+i^{\prime} \bar{a}-i^{\prime} b \\
=k \kappa \varphi z^{\prime}+i^{\prime} \tilde{a}_{3}, \quad \tilde{a}_{3} \in C_{*}(A), \\
\tilde{a}_{3}=\bar{a}-b .
\end{gathered}
$$

Due to (4) and (5) we realize that

$$
d l c_{2}^{\prime}-d c=d l c_{2}^{\prime}-k z \in C_{*}(A, A)
$$

hence we detect a $b_{1} \in C_{*}(A, A)$ such that

$$
d\left(l c_{2}^{\prime}-c-q_{\#} b_{1}\right)=0 .
$$

Because $l c_{2}^{\prime}-c-q_{\#} b_{1}$ is a cycle, there exist a $u^{\prime} \in C_{*}^{\prime}(X, A), \quad b_{2} \in C_{*}(A, A)$ satisfying

$$
\begin{gathered}
l u^{\prime}+q_{\#} b_{2}=l c_{2}^{\prime}-c+q_{\#} b_{1}-d w \\
w \in C_{*}(X, A), d u^{\prime} \in i m i^{\prime} .
\end{gathered}
$$


Defining $c^{\prime}=c_{2}^{\prime}-u^{\prime}$ we conclude with $a_{2}=b_{1}-b_{2}$

$$
\begin{array}{r}
l c^{\prime}+q_{\#} a_{2}=c+d w \\
d c^{\prime}=d c_{2}^{\prime}-d u^{\prime}=k \kappa \varphi z^{\prime}+i^{\prime} \tilde{a}_{3}-i^{\prime} a_{4}, \\
d u^{\prime}=i^{\prime} a_{4} \in i m i^{\prime} .
\end{array}
$$

With

$$
a_{3}=\tilde{a}_{3}-a_{4}
$$

we obtain

$$
d c^{\prime}=k \kappa \varphi z^{\prime}+i^{\prime} a_{3} .
$$

Implementing the chain homotopy of the beginning of this proof allows us to replace $\kappa \varphi z^{\prime}$ by $z^{\prime}$.

This completes the proof of the lemma.-

1.2. Lemma $\psi: H_{*}\left((K \otimes L)_{*}^{\prime \prime}\right)(X, A) \longrightarrow H_{*}(K \otimes L)(X, A)$ is epic (cf. [5] $\S 4, D 2))$. Proof: Suppose $z^{K}, z^{L}$ are cycles in $K_{p}(X, A)$ resp. $L_{q}(X, A)$, then we have relative cycles $z^{\prime K}, z^{\prime L}$ in $K^{\prime}{ }_{p}$ resp. $L^{\prime}{ }_{q}$ as well as chains $a^{K}, a^{L}$ in $K_{p}(A, A)$ resp. $L_{q}(A, A)$ such that

$$
\left\{\begin{array}{l}
z^{K} \sim l^{K}{z^{\prime}}^{K}+q_{\#} a^{K} \\
z^{L} \sim l^{L} z^{\prime L}+q_{\#} a^{L} .
\end{array}\right.
$$

Let us assume that we have identities (rather than homologies) in (8), then we deduce

$$
\begin{aligned}
z^{K} \otimes z^{L}= & l\left(z^{\prime K} \otimes z^{\prime L}\right)+q_{\#}\left(a^{K}\right) \otimes l^{L} z^{L}+l^{K} z^{\prime K} \otimes q_{\#}\left(a^{L}\right)+ \\
& +q_{\#}\left(a^{K} \otimes a^{L}\right)=l z^{\prime K \otimes L}+q_{\#}\left(a^{K \otimes L}\right),
\end{aligned}
$$

with $z^{\prime K \otimes L} \in(K \otimes L)_{*}^{\prime}(X, A), \quad a^{K \otimes L} \in(K \otimes L)_{*}(A, A)$.

Hence the homology class $\left\{z^{K} \otimes z^{L}\right\}$ is contained in im $\psi$.

Now it is easy to realize that the same conclusion holds in the general case (8).

Suppose that

$$
z^{K \otimes L}=\sum c_{i}^{K} \otimes c_{i}^{L}
$$

is any cycle in $(K \otimes L)_{*}(X, A)$, then $c_{i}^{K}, \quad c_{i}^{L}$ are either cycles in $K_{*}$ resp. $L_{*}$ or the summands are of the form $z_{i}^{K} \otimes c_{i}^{L}, d c_{i}^{L}=m z_{i}^{L}, m z_{i}^{K}=d c_{i}^{K}$, for $m \in \mathbb{Z}$. In this case we have

$$
d\left(z_{i}^{K} \otimes c_{i}^{L}+(-1)^{p-1} c_{i}^{K} \otimes z_{i}^{L}\right)=0 .
$$

Now we have due to lemma 1.1. (eventually replacing a cycle by a homologous one)

$$
\begin{gathered}
z_{i}^{K}=l^{K}{z^{\prime}}_{i}^{K}+q_{\#} b_{i}^{K}, \\
z_{i}^{L}=l^{L}{z^{\prime}}_{i}^{L}+q_{\#} b_{i}^{L}, \\
c_{i}^{K}=l^{K}{c^{\prime}}_{i}^{K}+q_{\#} a_{i}^{K},
\end{gathered}
$$




$$
c_{i}^{L}=l^{L} c_{i}^{\prime L}+q_{\#} a_{i}^{L}
$$

and therefore

$$
\begin{gathered}
z=z_{i}^{K} \otimes c_{i}^{L}+(-1)^{p-1} c_{i}^{K} \otimes z_{i}^{L}=l\left(z_{i}^{\prime K} \otimes c_{i}^{\prime L}\right)+(-1)^{p-1} l\left(c_{i}^{\prime K} \otimes z_{i}^{\prime L}\right)+ \\
+l\left(z_{i}^{\prime K} \otimes a_{i}^{L}+(-1)^{p-1} a_{i}^{K} \otimes z^{\prime L}+(-1)^{p-1} c_{i}^{\prime K} \otimes b_{i}^{L}\right)+ \\
+q_{\#}\left(b_{i}^{K} \otimes a_{i}^{L}\right)+(-1)^{p-1} q_{\#}\left(a_{i}^{K} \otimes b_{i}^{L}\right) .
\end{gathered}
$$

as a result

$$
z=l z^{\prime K \otimes L}+q_{\#} a^{K \otimes L}, \quad a^{K \otimes L} \in(K \otimes L)_{*}(A, A) .
$$

This completes the proof of lemma 1.2.-

1.3. Lemma: One has (cf. [5] $\S 4$ D3))

$$
\text { ker } \psi \subset \operatorname{ker} \bar{\partial} \text {. }
$$

Proof: Suppose we have

$$
d \sum c_{i}^{K} \otimes c_{i}^{L}=z^{K \otimes L} \in Z_{n}\left((K \otimes L)_{*}(X, A)\right),
$$

then we obtain

$$
z^{K \otimes L}=\sum d c_{i}^{K} \otimes c_{i}^{L}+(-1)^{p} c_{i}^{K} \otimes d c_{i}^{L} .
$$

Since $d c_{i}^{K}, d c_{i}^{L}$ are per definitionem bounding, application of lemma 1.1. yields the same situation as in the proof of the preceding lemma, but with all $b_{i}^{K}=b_{i}^{L}=0$. As a result the $q_{\#}\left(b_{i}^{K} \otimes a_{i}^{L}+(-1)^{p} a_{i}^{K} \otimes b_{i}^{L}\right)$ terms vanish and we establish

$$
z^{K \otimes L}=l z^{\prime K \otimes L} .
$$

This is equivalent to the assertion of the lemma.-

1.4. Lemma: $(K \otimes L)_{*}$ satisfies [5], §4] D2), hence there exists a

$$
\rho_{\#}: i m j_{\#} \longrightarrow(K \otimes L)_{*}^{\prime \prime}(X, A)
$$

such that $\psi \rho_{\# *}=1: i m j_{*} \longrightarrow i m j_{*}$ and

$$
p \kappa \simeq \rho_{\#} j_{\#} .
$$

Proof: We use the fact that 1) $\rho_{\#}^{K}, \rho_{\#}^{L}$ with the corresponding properties exist and 2) that D2) turns out to be equivalent to

$$
\operatorname{ker} j_{*} \subset \operatorname{ker} p_{*} \kappa \text {. }
$$

We form $\rho=\rho_{\#}^{K} \otimes \rho_{\#}^{L}: i m j_{\#} \longrightarrow(K \otimes L)_{*}^{\prime \prime}(X, A)$ which implies obviously (9).-

We summarize:

1.5. Proposition: The tensor product $\left((K \otimes L)_{*}, l, \kappa, \varphi, i^{\prime}\right)$ of two (irregular) 
chain functors is an irregular-chain-functor.

Proof: The crucial points of the proofs are the subject of the preceding lemmas, the remaining points (as [5] $\S 4$ D2), D4) to the extent in which they have not already been treated, cf. also definition 4.1) are trivial or immediate.

1.6. Proposition: Let $\lambda^{K}: \mathbf{K}_{*} \longrightarrow \tilde{\mathbf{K}}_{*}, \quad \lambda^{L}: \mathbf{L}_{*} \longrightarrow \tilde{\mathbf{L}}_{*}$ be mappings of chain functors, then

$$
\lambda^{K} \otimes \lambda^{L}:(\mathbf{K} \otimes \mathbf{L})_{*} \longrightarrow(\tilde{\mathbf{K}} \otimes \tilde{\mathbf{L}})_{*}
$$

furnishes a mapping of chain functors in a canonical way.

Proof: Follows from the definition of a mapping of chain functors (cf. [5] §4).-

1.7. Theorem: Let $\mathbf{K}_{*}, \mathbf{L}_{*}$ be two regular chain functors, then there exists for any $(X, A) \in \mathfrak{K}^{2}$ a Künneth formula which is natural in $(X, A)$ as well as in $\mathbf{K}_{*}$ and $\mathbf{L}_{*}$ :

$$
\begin{gathered}
0 \longrightarrow\left(H_{*}\left(\mathbf{K}_{*}\right)(X, A) \otimes H_{*}\left(\mathbf{L}_{*}\right)(X, A)\right)_{n} \longrightarrow H_{n}\left((\mathbf{K} \otimes \mathbf{L})_{*}(X, A) \longrightarrow\right. \\
\longrightarrow \operatorname{Tor}\left(H_{*}\left(\mathbf{K}_{*}\right)(X, A), H_{*}(\mathbf{L})(X, A)\right)_{n-1} \longrightarrow 0 .
\end{gathered}
$$

Proof: Since according to $\left(1^{\prime}\right)(K \otimes L)_{*}(\cdot) \approx K_{*}(\cdot) \otimes L_{*}(\cdot)$, this is a repetition of the proof of the Künneth formula for chain complexes (cf. [8]).

1.8. Proposition: The tensor product of two regular chain functors (resp. with compact carriers) is regular (resp. has compact carriers).

Proof: The fact that $(K \otimes L)_{*}(\quad)$ has compact carrier is an immediate consequence of the same property for $K_{*}(), L_{*}(\quad)$. In particular are $(K \otimes L)_{*}(\cdot)$ free with canonical base. Because $K_{*}(\cdot), L_{*}(\cdot)$ are free chain complexes, inclusions as well as $i^{\prime}, l$ (for non- irregular-chain-functors) induce monomorphisms onto direct summands.-

Let $f:(X, A) \longrightarrow(Y, B)$ be any mapping such that $H_{*}\left(\mathbf{K}_{*}\right)(f)=f_{* K}$ as well as $H_{*}\left(\mathbf{L}_{*}\right)(f)=f_{* L}$ is an isomorphism. Then we have a mapping between the two Künneth formulae for $(X, A)$ and $(Y, B)$. Since

$f_{* K} \otimes f_{* L}:\left(H_{*}\left(\mathbf{K}_{*}\right)(X, A) \otimes H_{*}\left(\mathbf{L}_{*}\right)(X, A)\right)_{n} \longrightarrow\left(H_{*}\left(\mathbf{K}_{*}\right)(Y, B) \otimes H_{*}\left(\mathbf{L}_{*}\right)(Y, B)\right)_{n}$

as well as the same expression for the torsion product are isomorphisms, and the Künneth formula is natural, we conclude that

$$
f_{*}=H_{n}\left((\mathbf{K} \otimes \mathbf{L})_{*}\right)(f): H_{n}\left((\mathbf{K} \otimes \mathbf{L})_{*}\right)(X, A) \longrightarrow H_{n}\left((\mathbf{K} \otimes \mathbf{L})_{*}\right)(Y, B)
$$

is an isomorphism.

Applying this to an excision map $(X, A) \longrightarrow(X / A, \star)$ assures us that the tensor product of two chain functors satisfies an excision property, whenever both factors do.

Since due to 1.5. $(\mathbf{K} \otimes \mathbf{L})_{*}$ are chain functors, 1.8. follows.-

1.9. Lemma: The tensor product of chain functors is up to an isomorphism associative and commutative:

$$
(\mathbf{K} \otimes \mathbf{L})_{*} \otimes \mathbf{M}_{*} \approx \mathbf{K}_{*} \otimes(\mathbf{L} \otimes \mathbf{M})_{*}
$$




$$
(\mathbf{K} \otimes \mathbf{L})_{*} \approx(\mathbf{L} \otimes \mathbf{K})_{*}
$$

Proof: This is an immediate consequence of the corresponding well-known fact for chain complexes.-

\section{Tensor products of spectra}

The (Boardman) category of spectra is defined in [1] or [13]. To each such spectrum $\mathbf{A}$ there exists a homology theory $\mathbf{A}_{*}$, defined on the subcategory $\mathfrak{P}^{2}$ of $\mathrm{CW}$ pairs.

According to [2] theorem 8.1. we obtain a (regular) chain functor ${ }^{A} \mathbf{C}_{*}$ such that the associated homology theory $H_{*}\left({ }^{A} \mathbf{C}_{*}\right)$ is isomorphic to $\mathbf{A}_{*}$.

This is true even if $\mathbf{A}_{*}$ is defined on Top ${ }^{2}$ and $\mathbf{A}$ not necessarily a CW spectrum. On the other hand there exists a functorial relationship between regular chain functors $\mathbf{C}_{*}$ and spectra $\left|\mathbf{C}_{*}\right|$, such that $H_{*}\left(\mathbf{C}_{*}\right) \approx\left|\mathbf{C}_{*}\right|_{*}$ (cf. [3] theorem 1.1.).

Let $\mathbf{A}, \quad \mathbf{B}$ be two spectra, then we define their tensor product by

$$
\mathbf{A} \otimes \mathbf{B}=\left|{ }^{A} \mathbf{C}_{*} \otimes{ }^{B} \mathbf{C}_{*}\right| .
$$

In order to ensure the independence of $\mathbf{A} \otimes \mathbf{B}$ of the choices of ${ }^{A} \mathbf{C}_{*},{ }^{B} \mathbf{C}_{*}$ we need:

2.1. Lemma: Suppose ${ }^{A} \mathbf{K}_{*},{ }^{A} \mathbf{L}_{*}\left({ }^{B} \mathbf{K}_{*},{ }^{B} \mathbf{L}_{*}\right)$ are two regular chain functors such that there exists a mapping of chain functors

$$
{ }^{A} \lambda:{ }^{A} \mathbf{K}_{*} \longrightarrow{ }^{A} \mathbf{L}_{*}\left({ }^{B} \lambda:{ }^{B} \mathbf{K}_{*} \longrightarrow{ }^{B} \mathbf{L}_{*}\right)
$$

inducing isomorphisms ${ }^{A} \lambda_{*}: H_{*}\left({ }^{A} \mathbf{K}_{*}\right) \approx H_{*}\left({ }^{A} \mathbf{L}_{*}\right)$ (resp. for ${ }^{B} \lambda_{*}$ ). Then

$$
\left({ }^{A} \lambda \otimes{ }^{B} \lambda\right)_{*}: H_{*}\left({ }^{A} \mathbf{K} \otimes{ }^{B} \mathbf{K}\right) \stackrel{\approx}{\longrightarrow} H_{*}\left({ }^{A} \mathbf{L} \otimes{ }^{B} \mathbf{L}\right)
$$

is an isomorphism of homology theories.

Proof: Observe that $\left({ }^{A} \lambda \otimes{ }^{B} \lambda\right)_{*}$ is a natural transformation of homology theories. We have short exact Künneth sequences

$\left.0 \rightarrow\left(H_{*}\left({ }^{A} \mathbf{K}\right)(\cdot) \otimes H_{*}\left({ }^{B} \mathbf{K}\right)(\cdot)\right)_{n} \rightarrow H_{n}\left({ }^{A} \mathbf{K} \otimes{ }^{B} \mathbf{K}\right)(\cdot) \rightarrow \operatorname{Tor}\left(H_{*}\left({ }^{A} \mathbf{K}\right)(\cdot), H_{*}\left({ }^{B} \mathbf{K}\right)(\cdot)\right)_{n-1}\right) \rightarrow 0$

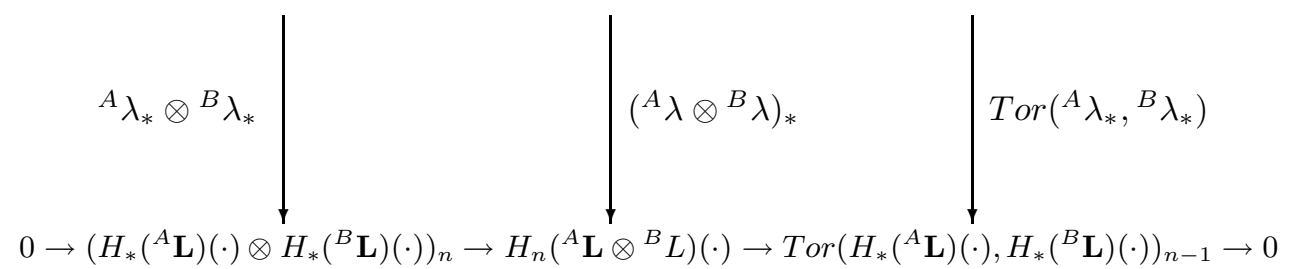

fitting into the commutative diagram with isomorphisms ${ }^{A} \lambda_{*} \otimes{ }^{B} \lambda_{*}, \operatorname{Tor}\left({ }^{A} \lambda_{*},{ }^{B} \lambda_{*}\right)$. Hence the third morphism $\left({ }^{A} \lambda \otimes{ }^{B} \lambda\right)_{*}$ is an isomorphism for any $(\cdot) \in \mathfrak{K}^{2}$. This completes the proof of the lemma.-

2.2. Lemma: Suppose ${ }^{A} \mathbf{K}_{*},{ }^{A} \mathbf{L}_{*}{ }^{B} \mathbf{K}_{*},{ }^{B} \mathbf{L}_{*}$ are as in 2.1. and assume that 
there exist chain transformations

$$
\left\{\begin{array}{l}
{ }^{A} \mathbf{K}_{*} \longrightarrow \cdot \longleftarrow \cdot \longleftrightarrow \cdots \cdots{ }^{A} \mathbf{L}_{*} \\
{ }^{B} \mathbf{K}_{*} \longrightarrow \cdot \longleftarrow \cdot{ }^{B} \mathbf{L}_{*}
\end{array}\right.
$$

(not necessarily of the same length) such that each transformation induces an isomorphism of the associated homology. Then there exists a chain of transformations

$$
\left({ }^{A} \mathbf{K} \otimes{ }^{B} \mathbf{K}\right)_{*} \longrightarrow \cdot \longleftarrow \cdots\left({ }^{A} \mathbf{L} \otimes{ }^{B} \mathbf{L}\right)_{*}
$$

such that each transformation induces an isomorphism on the homology level. Hence there exists a natural isomorphism

$$
\left({ }^{A} \mathbf{K} \otimes{ }^{B} \mathbf{K}\right)_{*} \approx\left({ }^{A} \mathbf{L} \otimes{ }^{B} \mathbf{L}\right)_{*} .
$$

Proof: We can without loss of generality assume that the chains in (2) are of equal length (by eventually inserting identities in one or the other direction). Application of lemma 2.1. to each seperate step yields the result.-

2.3. Lemma: Let ${ }^{A} \mathbf{K}_{*},{ }^{B} \mathbf{K}_{*},{ }^{A} \mathbf{L}_{*},{ }^{B} \mathbf{L}_{*}$ be chain functors such that ${ }^{A} \mathbf{K}_{*}$ and ${ }^{A} \mathbf{L}_{*}$ (resp. for B) yield isomorphic homology theories. Then there exists an isomorphism (3), which is induced by a chain of transformations of chain functors, each inducing an isomorphism of homology theories.

Proof: According to theorem 1.1. in [5] there exist two pairs of transformations of chain functors

$$
\begin{aligned}
& { }^{A} \mathbf{K}_{*} \longrightarrow \cdot \longleftarrow{ }^{A} \mathbf{L}_{*} \\
& { }^{B} \mathbf{K}_{*} \longrightarrow \cdot \longleftarrow{ }^{B} \mathbf{L}_{*}
\end{aligned}
$$

satisfying the assumption of 2.2. So we accomplish an isomorphism

$$
\left({ }^{A} \mathbf{K} \otimes{ }^{B} \mathbf{K}\right)_{*} \approx\left({ }^{A} \mathbf{L} \otimes{ }^{B} \mathbf{L}\right)_{*}
$$

which is induced by a chain of transformations of chain functors.-

2.4. Corollary: The tensor product $\mathbf{A} \otimes \mathbf{B}$ of two spectra $\mathbf{A}$ and $\mathbf{B}$ is determined by (1) up to an isomorphism of spectra in the Boardman homotopy category.

Proof: Since $|\cdot|$ is a functor such that $H_{*}\left(\mathbf{K}_{*}\right)(\cdot) \approx\left|\mathbf{K}_{*}\right|_{*}$, every transformation of chain functors $\lambda: \mathbf{K}_{*} \longrightarrow \mathbf{L}_{*}$, with $\lambda_{*}=$ isomorphism induces an isomorphism $\left|\mathbf{K}_{*}\right| \approx\left|\mathbf{L}_{*}\right|$ in the Boardman homotopy category (cf. [1] or [13]).) The assertion follows therefore from 2.3.-

Remark: Corollary 2.4. guarantees that, due to the main result of [5], the isomorphism class of $\mathbf{A} \otimes \mathbf{B}$ depends only on the isomorphism classes of the spectra $\mathbf{A}$ and $\mathbf{B}$, it does in particular not depend on the choices of the chain functors ${ }^{A} \mathbf{C}_{*}$ resp. ${ }^{B} \mathbf{C}_{*}$. Since there are many different ways to detect for a given spectrum $\mathbf{A}$ such a chain functor ${ }^{A} \mathbf{C}_{*}$ (cf. for example the remarks in [5] §5) this facts provides us with some freedom for the individual construction of a tensor product of two spectra. 
As an immediate corollary we deduce from 1.7., (1) and 2.4.:

2.5. Corollary: Let A, B be two spectra, then there exists a Kuenneth formula

$$
0 \longrightarrow\left(\mathbf{A}_{*}(\cdot) \otimes \mathbf{B}_{*}(\cdot)\right)_{\mathbf{n}} \longrightarrow\left(\mathbf{A} \otimes \mathbf{B}_{*}\right)(\cdot) \longrightarrow \operatorname{Tor}\left(\mathbf{A}_{*}(\cdot), \mathbf{B}_{*}(\cdot)\right)_{\mathbf{n}-\mathbf{1}} \longrightarrow \mathbf{0}
$$

which is natural in $(\cdot)$, as well as in $\mathbf{A}, \mathbf{B}$.

As a first application of this tensor product let $G_{1}, G_{2} \in \mathbf{A b}$, be abelian groups, $K\left(G_{i}\right)$ the associated Eilenberg-MacLane spectra, then we have:

2.6. Theorem: Suppose $\operatorname{Tor}\left(G_{1}, G_{2}\right)=0$, then there exists an isomorphism in the Boardman homotopy category

$$
K\left(G_{1} \otimes G_{2}\right) \approx K\left(G_{1}\right) \otimes K\left(G_{2}\right) .
$$

Proof: We deduce from 2.5. $(\operatorname{Tor}(\cdots, \cdots)=\cdots * \cdots)$

$$
\begin{gathered}
\left.0 \longrightarrow\left(K\left(G_{1}\right)_{*}(\cdot) \otimes K\left(G_{2}\right)_{*}(\cdot)\right)_{n} \longrightarrow\left(K\left(G_{1}\right) \otimes K\left(G_{2}\right)\right)\right)_{n}(\cdot) \longrightarrow \\
\left(K\left(G_{1}\right)_{*}(\cdot) * K\left(G_{2}\right)_{*}(\cdot)\right)_{n-1} \longrightarrow 0
\end{gathered}
$$

that the derived homology theory of $K\left(G_{1}\right) \otimes K\left(G_{2}\right)$ is an ordinary homology theory with $G_{1} \otimes G_{2}$ as coefficient group. Hence (1) follows.-

\section{An example}

We pointed out in $\S 1$ already that for irregular-chain-functors $K_{*}(X, A) \otimes$ $L_{*}(X, A)$ is not necessarily isomorphic to $K_{*}(X, A) \otimes L_{*}(X, A) \oplus K_{*}(X, A) \otimes$ $L_{*}(X) \oplus K_{*}(X) \otimes L_{*}(X, A)$. We display an irregular-chain-functor $\mathbb{Z}_{*}$ such that

$$
(\mathbf{K} \otimes \mathbb{Z})_{*} \approx \mathbf{K}_{*},
$$

for any chain functor $\mathbf{K}_{*}$ (cf. [5] $\S 4$ concerning the definition of equivalences of irregular-chain-functors).

We set

$$
\mathbb{Z}_{n}(X)=\left\{\begin{array}{l}
\left\langle z_{X}\right\rangle \mid \quad d z_{X}=0 \cdots n=0, \quad X \neq \emptyset \\
0 \cdots \text { elsewhere }
\end{array}\right.
$$

denoting by $\langle a\rangle$ the free abelian group generated by $a$, and

$$
\mathbb{Z}_{n}(X, A)=0 \quad \cdots A \neq \emptyset .
$$

Let $f:(X, A) \longrightarrow(Y, B)$ be a mapping, then we define either $f_{\#}\left(z_{X}\right)=z_{Y}$ or $f_{\#}=0$.

Moreover we set $\mathbb{Z}_{n}^{\prime}(X, A)=\mathbb{Z}_{n}(X), \quad \varphi\left(z_{X}\right)=z_{X}, \quad \kappa\left(z_{X}\right)=z_{X}$, whenever $X \neq \emptyset$

$$
l=\left\{\begin{array}{l}
i d \cdots A=\emptyset \\
0 \cdots A \neq \emptyset
\end{array}\right.
$$


This furnishes the structure of an irregular chain functor.

We have (for $X \neq \emptyset$, the remaining case is trivial)

$$
\operatorname{ker} \psi=\left\{\begin{array}{l}
0 \cdots A=\emptyset \\
0 \cdots A \neq \emptyset
\end{array} \subset \operatorname{ker} \bar{\partial}=\left\{\begin{array}{l}
\left\langle z_{X}\right\rangle \cdots A=\emptyset \\
0 \cdots A \neq \emptyset
\end{array}\right.\right.
$$

$\mathbb{Z}_{*}(X, X)$ is always acyclic, and $i^{\prime}\left(z_{A}\right)=z_{X}$, so that

$$
\begin{aligned}
& \kappa i_{\#}=i^{\prime} \\
& l=j_{\#} \varphi
\end{aligned}
$$

follows. In the same way we get $\operatorname{ker} j_{*} \subset \operatorname{ker} p_{*} \kappa_{*}$.

On the other hand $j_{\#}, \quad l$ are not inclusions.

This furnishes an irregular-chain-functor $\mathbb{Z}_{*}$.

We have for any chain functor $\mathbf{K}_{*}$

$$
\begin{gathered}
\left(K \otimes \mathbb{Z}_{*}(X, A)=K_{*}(X, A) \otimes \mathbb{Z}_{*}(X, A) \oplus K_{*}(X, A) \otimes \mathbb{Z}_{*}(X) \oplus\right. \\
\oplus K_{*}(X) \otimes \mathbb{Z}_{*}(X, A)=K_{*}(X, A),
\end{gathered}
$$

and

$$
\left\{\begin{array}{l}
(K \otimes \mathbb{Z})_{n}^{\prime}(X, A)=\bigoplus_{p+q=n} K_{p}^{\prime}(X, A) \otimes \mathbb{Z}_{q}^{\prime}(X, A) \oplus \\
\oplus \bigoplus_{p+q=n} K_{p}^{\prime}(X, A) \otimes \mathbb{Z}_{q}(A, A) \oplus \bigoplus_{p+q=n} K_{p}(A, A) \otimes \mathbb{Z}_{q}^{\prime}(X, A) \\
\approx K_{n}^{\prime}(X, A) \oplus K_{n}(A, A) .
\end{array}\right.
$$

The inclusion $\lambda: K_{*}(\cdot) \longrightarrow(K \otimes \mathbb{Z})_{*}(\cdot)$ which is defined by this procedure, is a mapping of (irregular) chain functors, i.e. compatible with the structure maps $i^{\prime}, l$ and, since $K_{*}(A, A)$ is acyclic and $(K \otimes \mathbb{Z})_{*}^{\prime} \supset K_{*}^{\prime}$, an equivalence of irregularchain-functors in the sense of definition 4.2. in [5]. To this end we have to observe that the sum (2) is an amalgamated sum in the sense of the definition of the $\otimes$ product of chain functors (cf. $\S 1$ ).

Moreover $\mathbb{Z}_{*}, \mathbb{Z}^{\prime}{ }_{*}$ have obviously compact carriers and satisfy a homotopy axiom $([5] \S 4 \mathrm{D} 4))$.

We have

$$
\begin{aligned}
& H_{n}\left(\mathbb{Z}_{*}\right)(X)=\left\{\begin{array}{l}
\mathbb{Z} \cdots X \neq \emptyset, n=0 \\
0 \cdots \text { elsewhere }
\end{array}\right. \\
& H_{n}\left(\mathbb{Z}_{*}\right)(X, A)=0 \cdots A \neq \emptyset, n \in \mathbb{Z}
\end{aligned}
$$

and summarize:

3.1. Theorem: There exists an irregular-chain-functor with compact carriers $\mathbb{Z}_{*}$ such that for any chain functor $\mathbf{K}_{*}$ there exists an equivalence of irregular-chainfunctors (cf. [5] definition 4.2)

$$
(\mathbf{K} \otimes \mathbb{Z})_{*} \approx \mathbf{K}_{*}
$$

. Remarks: 1) The chain functor $\mathbb{Z}_{*}$ a) does not induce monomorphisms for inclusions, b) does not admit a $l: \mathbb{Z}_{*}^{\prime}(X, A) \longrightarrow \mathbb{Z}_{*}(X, A)$ which is monic and c) does not turn all excision maps into isomorphisms (e.g. $\left.(X, \emptyset) \longrightarrow\left(X^{+}, \star\right), \quad X \neq \emptyset\right)$. 
So $\mathbb{Z}_{*}$ is not a regular chain functor in the sense of [5] definition 4.1.-

2) There can be no regular chain functor $\mathbf{C}_{*}$ such that

$$
(\mathbf{K} \otimes \mathbf{C})_{*} \approx \mathbf{K}_{*}
$$

for any regular chain functor $\mathbf{K}_{*}$ :

Assume to the contrary that such a $\mathbf{C}_{*}$ exists, then we would have a Künneth formula

$$
\begin{gathered}
0 \longrightarrow\left(H_{*}\left(\mathbf{K}_{*}\right)(\cdot) \otimes H_{*}\left(\mathbf{C}_{*}\right)(\cdot)\right)_{n} \longrightarrow H_{n}\left(\mathbf{K}_{*}(\cdot)\right) \longrightarrow \\
\operatorname{Tor}\left(H_{*}\left(\mathbf{K}_{*}\right)(\cdot), H_{*}\left(\mathbf{C}_{*}(\cdot)\right)_{n-1} \longrightarrow 0\right.
\end{gathered}
$$

implying that always

$$
H_{n}\left(\mathbf{C}_{*}\right)(\cdot)= \begin{cases}\mathbb{Z} \cdots & n=0 \\ 0 \cdots & n \neq 0 .\end{cases}
$$

Assume to this end that $H_{q}\left(\mathbf{C}_{*}\right) \neq 0, q \neq 0$, then we detect a $\mathbf{K}_{*}$ such that $H_{0}\left(\mathbf{K}_{*}\right)(\cdot)=0, H_{-q}\left(\mathbf{K}_{*}\right)(\cdot)=\mathbb{Z}$. Such a generalized homology theory clearly exists. This leads to a contradiction. Take on the other hand a $\mathbf{K}_{*}$ satisfying $H_{0}\left(\mathbf{K}_{*}\right)(\cdot)=$ $\mathbb{Z}$, then $H_{0}\left(\mathbf{C}_{*}\right)(\cdot)=\mathbb{Z}$ follows from the Künneth formula.

However (3) is impossible for a homology theory.

3) In particular there does not exist a spectrum $\boldsymbol{B}$ such that

$$
B \otimes E \approx E
$$

for every spectrum $\boldsymbol{E}$.

\section{Preparation of the localization process}

The localization process requires some general constructions which we display in this section:

Suppose $\mathfrak{L} \subset \mathfrak{K}^{2}$ is a subcategory, $\mathbf{C}_{*}$ a (regular or irregular) chain functor on $\mathfrak{K}^{2}, \quad \mathbf{K}_{*}$ a (regular or irregular) chain functor on $\mathfrak{L}$. Since we do not know anything about $\mathfrak{L}$ (it may not contain any compact objects at all) it does not make sense to assume that $\mathbf{K}_{*}$ has compact carriers. Let $\alpha: \mathbf{K}_{*} \longrightarrow \mathbf{C}_{*} \mid \mathfrak{L}$ be a g-transformation of chain functors (cf. [5] definition 4.3.).

4.1. Theorem There exists a chain functor $\overline{\mathbf{K}}_{*}$ on $\mathfrak{K}^{2}$, mappings of chain functors $\tau: \mathbf{K}_{*} \longrightarrow \overline{\mathbf{K}}_{*} \mid \mathfrak{L}, \bar{\alpha}: \overline{\mathbf{K}}_{*} \longrightarrow \mathbf{C}_{*}$ such that

1) $(\bar{\alpha} \mid \mathfrak{L}) \tau=\alpha$, and $\tau: \mathbf{K}_{*} \subset \overline{\mathbf{K}}_{*} \mid \mathfrak{L}$ is a strong deformation retract.

2) For any g-transformation $\beta: \mathbf{L}_{*} \longrightarrow \mathbf{C}_{*}, \mathbf{L}_{*}$ any chain functor on $\mathfrak{K}^{2}$,

$\gamma: L_{*} \mid \mathfrak{L} \longrightarrow K_{*}$, satisfying $(\beta \mid \mathfrak{L})=\alpha \gamma$, there exists a up to chain homotopy unique $r: \mathbf{L}_{*} \longrightarrow \overline{\mathbf{K}}_{*}$ such that $\bar{\alpha} r=\beta, r \mid \mathfrak{L}=\tau \gamma$

3) If $\mathbf{K}_{*}, \mathbf{C}_{*}$ are regular, then $l, i^{\prime},((X, A) \subset(Y, B))_{\#}$ for $\mathbf{K}_{*}$ are inclusions onto direct summands (cf. [5] §4 D3) *)); if all involved chain functors have compact carriers, then $\overline{\mathbf{K}}_{*}$ can be assumed to have compact carriers.

Proof: The proof is a slight adaptation of the classical way to accomplish Kan extensions. 
I) Assume we are dealing not with chain functors $\mathbf{C}_{*}, \mathbf{K}_{*}$ but merely with functors $C_{*}: \mathfrak{K}^{2} \longrightarrow \mathbf{c h}$ (=category of free chain complexes with natural bases), $K_{*}: \mathfrak{K}^{2} \longrightarrow \mathbf{c h}$ (without the additional structure of chain functors). Suppose $a \in C_{*}(X, A), \quad(X, A) \in \mathfrak{K}^{2}$, then we consider collections

$$
\mathbf{a}=\left\{a ; a_{f} \in K_{*}(E, F) \mid f:(X, A) \longrightarrow(E, F) \in \mathfrak{L}\right\}
$$

satisfying

$$
a_{g f}=g_{\#} a_{f}, \quad g \in \mathfrak{L}\left((E, F),\left(E^{\prime}, F^{\prime}\right)\right)
$$

and

$$
f_{\#}(a)=\alpha\left(a_{f}\right)
$$

We set

$$
\hat{K}_{n}(X, A)=\left\langle\left\{\mathbf{a} \mid a \in C_{n}(X, A)\right\}\right\rangle
$$

$\langle\cdots\rangle$ denoting the abelian group generated by $\cdots$. If $h \in \mathfrak{K}^{2}((X, A),(Y, B))$, we define

$$
h_{\#}(\mathbf{a})=\left\{h_{\#}(a) ; a_{f h} \mid f \in \mathfrak{K}^{2}((Y, B),(E, F)), \quad(E, F) \in \mathfrak{L}\right\},
$$

and

$$
d \mathbf{a}=\left\{d a ; d a_{f}\right\}
$$

Assuming that $\mathbf{C}_{*}$ has compact carrier, then, by a well-known technique we equip $\hat{K}_{*}: \mathfrak{K}^{2} \longrightarrow$ ch with compact carriers: We perform the previous construction only for compact $(X, A)$ and form the direct limit.

So $\hat{K}_{*}: \mathfrak{K}^{2} \longrightarrow \mathbf{c h}$ becomes a functor with compact carrier, whenever $K_{*}$ and $C_{*}$ have this property.

Setting $\bar{\alpha}(\mathbf{a})=a$, yields a natural transformation $\bar{\alpha}: \hat{K}_{*} \longrightarrow C_{*}$.

We set

$$
\begin{aligned}
& \tau: \mathbf{K}_{*} \longrightarrow \hat{\mathbf{K}}_{*} \mid \mathfrak{L} \\
& c \longmapsto\left\{\alpha(c) ; f_{\#}(c)\right\}
\end{aligned}
$$

$c$ in a base of $\mathbf{K}_{*}(X, A), \quad(X, A) \in \mathfrak{L}$ and for given $\beta: \mathbf{L}_{*} \longrightarrow \mathbf{K}_{*}$ as in the theorem:

$$
\begin{gathered}
r: \quad \mathbf{L}_{*} \longrightarrow \hat{\mathbf{K}}_{*} \\
c \longmapsto\left\{\beta(c) ; \gamma f_{\#}(c)\right\} .
\end{gathered}
$$

Since $K_{*}(X, A)$ has a natural base, $\tau, \beta$ are natural. We deduce

$$
\bar{\alpha} \tau(c)=\bar{\alpha}\left\{\alpha(c) ; f_{\#}(c)\right\}=\alpha(c)
$$

and

$$
\begin{array}{cc}
\bar{\alpha} r(c)=\beta(c), & c \in L_{*}(X, A), \\
\tau \gamma(c)=\left\{\alpha \gamma(c) ; f_{\#} \gamma(c)\right\}=\left\{\beta(c) ; \gamma f_{\#}(c)\right\}
\end{array}
$$


$c \in L_{*}(E, F), \quad f:(E, F) \longrightarrow\left(E^{\prime}, F^{\prime}\right)$ in $\mathfrak{L}$.

Moreover for $\left(E^{\prime}, F^{\prime}\right),(X, A) \in \mathfrak{L}, f:(X, A) \longrightarrow\left(E^{\prime}, F^{\prime}\right)$, we have a

$$
\begin{aligned}
\hat{K}_{*}(X, A) & \longrightarrow K_{*}(X, A) \\
\left\{a ; a_{f}\right\} & \longmapsto \quad a_{1}, \quad 1=1_{(X, A)}
\end{aligned}
$$

and observe that in this case $\tau \mid \mathfrak{L}$ is an isomorphism.

We have a subcomplex $K_{n}^{0}(X, A) \subset \hat{K}_{n}(X, A)$ consisting of all those a with $a=$ $\bar{\alpha}(\mathbf{a})=0$. Now we define

$$
\bar{K}_{*}(X, A)=\hat{K}_{*}(X, A) \cup \text { cone } K_{*}^{0}(X, A) .
$$

The mappings $\bar{\alpha}, r$ an be extended over $\bar{K}_{n}$. We will deal with $\tau: \mathbf{K}_{*} \longrightarrow \overline{\mathbf{K}}_{*} \mid \mathfrak{L}$ at the end of II) of the present proof. Unlike for $\hat{K}_{n}$ we have a uniqueness assertion for $r$ :

Suppose $r, \tilde{r}: L_{*} \longrightarrow \bar{K}_{*}$ be such that $\bar{\alpha} r=\bar{\alpha} \tilde{r}$, then we deduce $r \simeq \tilde{r}$.

Proof: If $\tilde{r}(c)=\left\{a ; a_{f}\right\}$, then $\bar{\alpha} \tilde{r}(c)=a=\beta(c)$ implies $\tilde{r}(c)=\left\{\beta(c) ; a_{f}\right\}$, hence $\tilde{r}(c)-r(c)=\left\{0 ; a_{f}-f_{\#}(c)\right\} \in K_{n}^{0}$, so that the existence of a chain homotopy $r \simeq \tilde{r}$ follows.

II) We are now obliged to endow $\bar{K}_{*}$ with the structure of a chain functor. This is in a first step accomplished for $\hat{K}_{*}$, followed by attaching the cone over $K_{*}^{0}$ afterwards. In detail we have

1) to establish non-natural mappings $\varphi, \kappa$ together with chain homotopies $D_{1}$ : $\varphi \kappa \simeq 1, D_{2}: j_{\#} \varphi \simeq l$, such that the following additional conditions hold for $C_{*}=\hat{K}_{*}$ :

2) Every cycle $z \in Z_{n}\left(C_{*}(X, A)\right)$ is associated with a $x \in C_{n+1}(X, A), \quad z^{\prime} \in$ $C_{n}^{\prime}(X, A), \quad d z^{\prime} \in i m i^{\prime}, \quad a \in C_{n}(A, A)$ such that

$$
l z^{\prime}+q_{\#} a=z+d x
$$

3) $\operatorname{ker} \psi \subset \operatorname{ker} \bar{\partial}\left(\operatorname{cf}\right.$. [5] $\S 4, \psi: H_{*}\left(C_{*}^{\prime \prime}(X, A)\right) \longrightarrow H_{*}\left(C_{*}(X, A)\right), \quad C_{*}^{\prime \prime}(X, A)=$ $\left.C_{*}^{\prime}(X, A) / i m i^{\prime}\right)$

4) $\psi$ is epic; ker $j_{*} \subset$ ker $p_{*} \kappa, \quad p: C_{*}^{\prime}(X, A) \longrightarrow C_{*}^{\prime \prime}(X, A)$;

$5)$ every cycle $z \in Z_{n}\left(C_{*}(X, X)\right)$ is bounding: $d x=z$.

6) Let $H: f_{0} \simeq f_{1}:(X, A) \longrightarrow(Y, B)$ be a homotopy, then we have a chain homotopy $\chi: C_{n}(X, A) \longrightarrow C_{n+1}(Y, B)$, satisfying $d \chi(a)+\chi d a=f_{0 \#}(a)-f_{1 \#}(a)$, (Property D4) in the definition of a chain functor [5] §4.)

All these objectives are achieved by a modification of the procedure in I), which we explain in the case of 1 ):

To that end assume that $C_{*}, K_{*}$ are endowed with the structure of chain functors and that $\alpha$ is a mapping of chain functors. We change the definition of (1) in the following way:

Instead of using continuous mappings $f \in \mathfrak{K}^{2}$ we introduce formal arrows $(X, A) \stackrel{\varphi}{\longrightarrow}$ $X, X \stackrel{\kappa}{\longrightarrow}(X, A)$ and substitute for $f$ words

$$
(X, A) \stackrel{k_{1}}{\longrightarrow} \cdot \stackrel{k_{2}}{\longrightarrow} \cdot \cdots \stackrel{k_{n}}{\longrightarrow}(E, F)
$$


where each $k_{i}$ is either of the form $\varphi$ or $\kappa$ or a continuous mapping, whereas $\varphi$ can only appear whenever the image of $a$ under the word $(X, A) \longrightarrow \cdots \longrightarrow\left(X_{1}, A_{1}\right)$ lies in $C_{n}^{\prime}\left(X_{1}, A_{1}\right)$.

Condition (2) in I) is replaced by the same one, but now words (5) replacing continuous mappings $f$, while $g$ is still assumed to be continuous. There are no relations between the letters $\varphi, \kappa$ or between one of these and continuous mappings. So (2) takes the form

$$
a_{g w}=g_{\#} a_{w},
$$

where $w$ is any suitable word and $g$ is continuous.

Condition (3) reads now:

$$
w_{\#}(a)=\alpha\left(a_{w}\right) .
$$

We set

$$
\hat{K}_{n}^{\prime}(X, A)=\left\langle\left\{\mathbf{a} \in \hat{K}_{n}(X, A) \mid a \in C_{n}^{\prime}(X, A)\right\}\right\rangle
$$

resp. for $\bar{K}_{*}$, and detect a natural inclusion induced by $l$ whenever $C_{*}, K_{*}$ are regular,

$$
l: \bar{K}_{*}^{\prime}(X, A) \subset \bar{K}_{*}(X, A) .
$$

We define

$$
\begin{aligned}
\varphi(\mathbf{a}) & =\left\{\varphi(a) ; a_{w \varphi}\right\} \\
\kappa(\mathbf{a}) & =\left\{\kappa(a) ; a_{w \kappa}\right\},
\end{aligned}
$$

for suitable words $w: X \longrightarrow(E, F)$ resp. $w:(X, A) \longrightarrow(E, F)$ of the form (5), realizing that this provides us with elements in $\hat{K}_{*}$. Observe that we still have

$$
d\left\{a ; a_{w}\right\}=\left\{d a ; d a_{w}\right\}
$$

Finally setting

$$
i^{\prime}=\kappa i_{\#}
$$

ensures that $\bar{\alpha}$ is compatible with $\varphi, \kappa, i^{\prime}, l$.

In a next step we have to establish chain homotopies $D_{1}: \varphi \kappa \simeq 1, D_{2}: j_{\#} \varphi \simeq l$ and other relations 2)-5) which are required of a chain functor. This is in all cases accomplished by translating the specific problem into that of finding suitable (with the exception of $\chi$, non- natural) mappings $\delta_{1}, \delta_{2}, \eta, \gamma_{1}, \gamma_{2}, \delta$, $\chi$, which in turn is finally solved in the same way as we established $\kappa$ and $\varphi$ before.

Let us start with 5):

By choosing a basis for the free abelian group $Z_{n}\left(C_{*}(X, X)\right)$ of all cycles in $C_{*}(X, X)$ we find a homomorphism $\delta: Z_{n}\left(C_{*}(X, X)\right) \longrightarrow C_{n+1}(X, X)$ satisfying $d \delta(z)=z$. Again we form words as in (5) but now also incorporating formal arrows of the form $\delta$, finally obtaining a $\delta: Z_{n}\left(\hat{K}_{*}(X, X)\right) \longrightarrow \hat{K}_{n+1}(X, X)$ guaranteeing that $\hat{K}_{*}(X, X)$ and $\bar{K}_{*}(X, X)$ are acyclic.

We have for any $\mathbf{a}=\left\{a ; a_{w}\right\} \in \hat{K}_{*}(X, X)$

$$
\begin{gathered}
\delta \mathbf{a}=\left\{\delta a ; a_{w \delta}\right\} \\
d \delta \mathbf{a}=\mathbf{a} .
\end{gathered}
$$


Here for the first time we encounter a case where $d\left\{a ; a_{w}\right\}=\left\{d a ; \tilde{a}_{w}\right\} \neq\left\{d a ; d a_{w}\right\}$. The homotopy $D_{1}: \varphi \kappa \simeq 1$ is associated with a mapping $\delta_{1}: C_{n}(X) \longrightarrow C_{n+1}(X)$ which again is established by using the fact that all occuring abelian groups are free, satisfying $d \delta_{1}(a)+\delta_{1}(d a)=\varphi \kappa(a)-a$.

We introduce a new arrow $\delta_{1}: X \longrightarrow X$ and define for $\mathbf{a}=\left\{a, a_{w}\right\}$

$$
\delta_{1}(\mathbf{a})=\left\{\delta_{1}(a) ; a_{w \delta_{1}}\right\},
$$

confirming that $\delta_{1} \mathbf{a}$ is well-defined in $\hat{K}_{*}$. Suppose $d \hat{a}=\left\{d a ; \tilde{a}_{w}\right\}$, then we set

$$
d \delta_{1} \mathbf{a}=\left\{\delta_{1} a ; a_{w \varphi \kappa}-a_{w}-\tilde{a}_{w \delta_{1}}\right\} .
$$

Because

$$
\delta_{1} d \mathbf{a}=\left\{\delta_{1} d a ; \hat{a}_{w \delta_{1}}\right\}
$$

we have

$$
d \delta_{1} \mathbf{a}+\delta_{1} d \mathbf{a}=\varphi \kappa \mathbf{a}-\mathbf{a} .
$$

The chain homotopy $D_{2}: j \varphi \simeq l$ is treated in the same way Dealing with 2) we have to translate this into a mapping

$$
\begin{aligned}
\eta: Z_{n}\left(C_{*}(X, A)\right) & \longrightarrow C_{n}^{\prime}(X, A) \oplus C_{n+1}(X, A) \oplus C_{n-1}(A) \\
z & \longmapsto\left(\eta^{\prime}(z), \eta^{\prime \prime}(z), \eta^{\prime \prime \prime}(z)\right)
\end{aligned}
$$

satisfying

$$
\begin{array}{r}
d \eta^{\prime \prime}(z)+z=l \eta^{\prime}(z)+q_{\#} \delta s_{\#} \eta^{\prime \prime \prime}(z) \\
d \eta^{\prime}(z)=i^{\prime} \eta^{\prime \prime \prime}(z) .
\end{array}
$$

So we introduce three arrows $\eta^{\prime}, \eta^{\prime \prime}, \eta^{\prime \prime \prime}$ :

$$
\eta^{(k)} \mathbf{a}=\left\{\eta^{(k)}(a) ; a_{w \eta^{(k)}}\right\},
$$

with the following boundary relations:

$$
\begin{gathered}
d \eta^{\prime} \mathbf{a}=\left\{d \eta^{\prime} a ; a_{w \eta^{\prime \prime \prime} \kappa i}\right\} \\
d \eta^{\prime \prime} \mathbf{a}=\left\{d \eta^{\prime \prime} a ; a_{w \eta^{\prime}}+a_{w \eta^{\prime \prime \prime} s \delta q}-a_{w}\right\} \\
d \eta^{\prime \prime \prime} \mathbf{a}=0 .
\end{gathered}
$$

As a result we obtain for $\mathbf{a} \in Z_{n}\left(\hat{K}_{*}(X, A)\right)$

$$
d \eta^{\prime \prime} \mathbf{a}+\mathbf{a}=\eta^{\prime} \mathbf{a}+q_{\#} \delta s_{\#} \eta^{\prime \prime \prime} a .
$$

Condition 3 ) is easily translated into a mapping

$$
\gamma_{1}: B_{n}\left(C_{*}(X, A)\right) \longrightarrow C_{n}(A)
$$

( $B_{n}=$ bounding cycles) such that $d \gamma_{1}(z)=d \eta^{\prime}(z)$.

By the same procedure we associate 4$)$ with a mapping and ultimately with a suitable arrow $\gamma_{2}$.

We arrive at 6): 
For any chain functor, a homotopy $H: i_{0} \simeq i_{1}:(X, A) \subset(X, A) \times I$ determines a chain homotopy

$$
\chi: C_{n}(X, A) \longrightarrow C_{n+1}((X, A) \times I)
$$

satisfying

$$
d \chi(a)+\chi(d a)=i_{0 \#}(a)-i_{1 \#}(a) .
$$

We invent an arrow $\chi$ and set as before $\chi \mathbf{a}=\left\{\chi a ; a_{w \chi}\right.$. If $d \mathbf{a}=\left\{d a ; \tilde{a}_{w}\right\}$, we define

$$
d \chi \mathbf{a}=\left\{d \chi a ; w_{w i_{0}}-a_{w i_{1}}-\tilde{a}_{w \chi}\right\}
$$

accomplishing a chain homotopy

$$
d \chi \mathbf{a}+\chi d \mathbf{a}=i_{0 \#} \mathbf{a}-i_{1 \#} \mathbf{a} .
$$

Since every homotopy $\tilde{H}: f_{0} \simeq f_{1}:(X, A) \longrightarrow(Y, B), \quad \tilde{H}:(X, A) \times I \longrightarrow(Y, B)$ is associated with a

$$
\begin{gathered}
\tilde{\chi}: C_{n}(X, A) \longrightarrow C_{n+1}(Y, B), \\
\tilde{\chi}=\tilde{H} \chi,
\end{gathered}
$$

we obtain a word $\tilde{\chi}=\tilde{H} \chi$ furnishing a chain homotopy

$$
d \tilde{\chi} \mathbf{a}+\tilde{\chi} d \mathbf{a}=f_{0 \#} \mathbf{a}-f_{1 \#} \mathbf{a} .
$$

The final $\tilde{K}_{*}$ is now constructed by the devices of I) but with words involving all arrows $\varphi, \kappa, \delta, \delta_{1}, \delta_{2}, \eta, \gamma_{1}, \gamma_{2}, \delta, \chi$ together with continuous mappings as letters. Since the induced chain homotopies are natural, cf. [5] D4), we have to assume that $\tilde{\chi}$ commutes appropriately with continuous mappings. By construction this yields a chain functor, such that $\bar{\alpha}$ becomes a mapping of chain functors.

We form $K_{*}^{0}(X, A)$ and $\bar{K}_{*}(X, A)=\hat{K}_{*}(X, A) \cup \operatorname{cone}\left(K_{*}^{0}(X, A)\right.$ as in I $)$. This yields a chain functor; $\bar{\alpha}$ is immediately extended over $\bar{K}_{*}$.

Since $\alpha$ and $\beta$ are supposed to be g-transformations, we can assume without loss of generality, i.e. up to isomorphisms (cf. [5] 4.4.), that they commute stricly with all words $w_{\#}$.

Let $\beta: \mathbf{L}_{*} \longrightarrow \mathbf{C}_{*}$ be as in the theorem, and set $\tilde{r}(c)=\left\{\beta(c) ; \gamma w_{\#}(c)\right\} \in \hat{K}_{*}(X, A)$ where $c \in L_{*}(X, A)$ observing that

$$
\tilde{r}(d c)-d \tilde{r}(c) \in K_{*}^{0}(X, A)
$$

and

$$
\bar{\alpha} \tilde{r}=\beta
$$

so that there exists a $D(c) \in \bar{K}_{*}(X, A)$ with

$$
d D(c)+D(d c)=\tilde{r}(d c)-d \tilde{r}(c) .
$$

Hence $r(c)=\tilde{r}(c)+D(c)$ is a chain mapping, satisfying $\bar{\alpha} r=\beta$.

We define on the subcategory $\mathfrak{L}$ :

$$
\begin{aligned}
\hat{\tau}: \mathbf{K}_{*} & \longrightarrow \hat{\mathbf{K}}_{*} \mid \mathfrak{L} \\
b & \longmapsto\left\{\alpha(b) ; w_{\#}(b)\right\}
\end{aligned}
$$


and

$$
\begin{aligned}
\hat{\tau}_{0}: \hat{\mathbf{K}}_{*} \mid \mathfrak{L} & \longrightarrow \mathbf{K}_{*} \\
\left\{a ; a_{w}\right\} & \longmapsto a_{1} .
\end{aligned}
$$

We calculate

$$
\begin{gathered}
w_{\#} \alpha(b)=\alpha w_{\#}(b) \\
g_{\#} w_{\#}(b)=(g w)_{\#}(b) \\
\hat{\tau}_{0} \hat{\tau}(b)=b, \\
\hat{\tau} \hat{\tau}_{0}\left\{a ; a_{w}\right\}=\hat{\tau}\left(a_{1}\right)=\left\{\alpha\left(a_{1}\right) ; w_{\#} a_{1}\right\}=\left\{a ; w_{\#} a_{1}\right\} .
\end{gathered}
$$

Since $\hat{\tau} \hat{\tau}_{0}\left\{a ; a_{w}\right\}-\left\{a ; a_{w}\right\} \in \hat{\mathbf{K}}_{*}^{0}$, the existence of a homotopy $\tau \tau_{0} \simeq 1$ follows for the corresponding

$$
\begin{gathered}
\tau: \mathbf{K}_{*} \longrightarrow \overline{\mathbf{K}}_{*} \mid \mathfrak{L} \\
\tau_{0}: \overline{\mathbf{K}}_{*} \mid \mathfrak{L} \longrightarrow \mathbf{K}_{*},
\end{gathered}
$$

which are easily established, turning $\hat{\mathbf{K}}_{*}$ into a strong deformation retract of $\mathbf{K}_{*}$. This can easily be extended to a transformation $\tau: \mathbf{K}_{*} \longrightarrow \overline{\mathbf{K}}_{*}$, displaying the same property.

One has $r \mid \mathfrak{L}=\tau \gamma$. All transformations $\bar{\alpha}, r, \tau$ commute with $l$ and $i^{\prime}$, so turning out to be mappings of chain functors. All $\hat{K}_{*}\left(\right.$ ) and of course all $\bar{K}_{*}()$ are free chain complexes with natural bases.

Suppose that $\mathbf{K}_{*}, \mathbf{C}_{*}$ are regular: Since $l, i^{\prime}$ and $((X, A) \subset(Y, B))_{\#}$ are monomorphisms onto direct summands for $\mathbf{K}_{*}$, the same holds clearly for $\hat{\mathbf{K}}_{*}$ and this carries immediately over to $\overline{\mathbf{K}}_{*}$. As in the course of I), we can assume that $\hat{K}_{*}$, hence $\bar{K}_{*}$ have compact carriers, whenever $\mathbf{K}_{*}$ has.

This settles the assertion 3) of the theorem.

The universality of $\overline{\mathbf{K}}_{*}, \bar{\alpha}$ follows as in I).

This completes the proof of theorem 4.1.-

4.2. Corollary: Under the same assumptions as in 4.1. let $\bar{\alpha}^{i}: \bar{K}_{*}^{(i)} \longrightarrow C_{*}$, $r^{i}: L_{*} \longrightarrow \bar{K}_{*}^{(i)}, \quad i=1,2$ be two solutions, then there exists a natural chain homotopy $\bar{K}_{*}^{(1)} \simeq \bar{K}_{*}^{(2)}$ commuting with $\bar{\alpha}^{i}$, $r^{i}$ up to homotopy. Proof: Follows immediately from the uniqueness assertion (up to homotopy) in 4.1.-

One important application of the preceding assertion deals with the case that $\mathfrak{L}$ consists of a single object $E \in \mathfrak{K}$ ( with respect to which one is going to localize a chain functor resp. a spectrum), with the identity as the only morphism in $\mathfrak{L}$.

In addition we need some simple facts about chain complexes, which are presumably well-known:

4.3. Lemma: Let $C_{*}$ be any chain complex, then there exists an acyclic chain complex $U_{*}$ and a chain mapping $\alpha: U_{*} \longrightarrow C_{*}$ such that for any chain mapping $\beta: V_{*} \longrightarrow C_{*}, \quad V_{*}$ acyclic, there exists a chain mapping $\rho: V_{*} \longrightarrow U_{*}$, such that 
$\alpha \rho=\beta$. Proof: Let $D_{*}$ be the chain complex

$$
D_{0}=\langle x\rangle, \quad D_{-1}=\langle d x\rangle, \quad D_{n}=0, n \neq 0,1
$$

$(\langle\cdots\rangle=$ free abelian group generated by $\cdots)$.

We can take for $U_{*}$ the chain complex $D_{*} \otimes C_{*}$ and observe that:

1) $D_{*} \otimes C_{*}$ is acyclic.

2) $\alpha: D_{*} \otimes C_{*} \longrightarrow C_{*}, \alpha(x \otimes c)=c, \alpha(d x \otimes c)=0$ is a chain mapping

3) Let $\beta: V_{*} \longrightarrow C_{*}$ be a chain mapping, then we have $1_{D_{*}} \otimes \beta: D_{*} \otimes V_{*} \longrightarrow$ $D_{*} \otimes C_{*}$ and since $V_{*}$ is acyclic, there exists a chain mapping $\gamma: V_{*} \longrightarrow D_{*} \otimes V_{*}$ such that

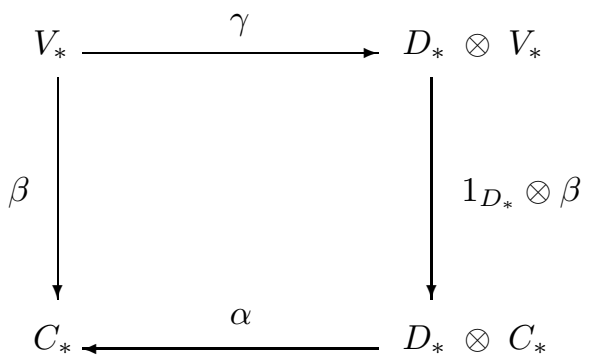

is commutative, so that we are allowed to set

$$
\rho=\left(1_{D_{*}} \otimes \beta\right) \gamma
$$

Only 3) needs proof:

We establish $\gamma$ at first on the cycles $z \in V_{n}$ : Since $V_{*}$ is acyclic there exists a $\bar{z} \in V_{n+1}$ such that $d \bar{z}=z$. We set

$$
\gamma(z)=z \otimes x+(-1)^{n+1} \bar{z} \otimes d x
$$

so that $d \gamma(z)=0$. Let $c \in V_{n}$ be any chain, then we find $\overline{d c}$ and $\bar{c} \in V_{n+1}$ such that $d \bar{c}=c-\overline{d c}$, and set $\gamma(c)=c \otimes x+(-1)^{n+1} \bar{c} \otimes d x$, so that

$$
\begin{gathered}
d \gamma(c)=d c \otimes x+(-1)^{n} c \otimes d x+(-1)^{n+1} d \bar{c} \otimes d x \\
=d c \otimes x+(-1)^{n} c \otimes d x+(-1)^{n+1} c \otimes d x+(-1)^{n} \overline{d c} \otimes d x=\gamma(d c) .
\end{gathered}
$$

The commutativity of (6) is immediate.-

Suppose we fix a subcategory $\mathfrak{L} \subset \mathfrak{K}^{2}$ and to each $(K, L) \in \mathfrak{L}$ a $U_{*}(K, L)=$ $U_{*}\left(A_{*}(K, L)\right)$ as in 4.3. The fact that $U_{*}\left(C_{*}\right)=D_{*} \otimes C_{*}$ ensures that the construction of $U_{*}$ as well as that of $\alpha$ is functorial and inherits the structure of a chain functor from the given chain functor $\mathbf{A}_{*}$. Moreover the mapping $\alpha$ is a $\mathrm{g}$ transformation of chain functors (cf. [5] 4.3.).

In order to transfer 4.3. from chain complexes to chain functors, we need:

4.4. Definition: Suppose a chain functor $\mathbf{A}_{*}$ is defined on a category $\mathfrak{K}^{2}$ and $\mathfrak{L} \subset \mathfrak{K}^{2}$ is a given subcategory. $\mathbf{A}_{*}$ is $\mathfrak{L}$-acyclic whenever there exists a natural chain homotopy $D$ between the identity $1: \mathbf{A}_{*}\left|\mathfrak{L} \longrightarrow \mathbf{A}_{*}\right| \mathfrak{L}$ and the trivial map, i.e. 
one has

$$
d D(c)+D(d c)=c, \quad c \in A_{*}(K, L),(K, L) \in \mathfrak{L},
$$

and

$$
D(c) \in A_{*}^{\prime}
$$

whenever $c \in A_{*}^{\prime}$.

This makes sense also if $\mathfrak{L}=\mathfrak{K}^{2}$. In general $\mathfrak{L}$-acyclicity is stronger than the mere requirement that $H_{*}\left(\mathbf{A}_{*} \mid \mathfrak{L}\right)=0$. however ist is easy to realize that in some cases both concepts coincide:

1) Suppose $\mathfrak{L}$ consists of one single object $E$ with no morphisms but the identity.

2) $\mathfrak{L}$ is a tower $L_{1} \longrightarrow L_{2} \longrightarrow \cdots$

$3) \mathfrak{L}$ is any category and $\mathbf{A}_{*}=$ cone $\mathbf{B}_{*}$ for some chain functor $\mathbf{B}_{*}$.

This allows us to assert:

4.5. Lemma: Let $\mathbf{A}_{*}$ be a chain functor on $\mathfrak{K}^{2}, \mathfrak{L} \subset \mathfrak{K}^{2}$ a subcategory, then there exists an $\mathfrak{L}$-acyclic chain functor $\mathbf{U}_{*}$ on $\mathfrak{L}$, a g-transformation of chain functors $\alpha: \mathbf{U}_{*} \longrightarrow \mathbf{A}_{*} \mid \mathfrak{L}$, such that for any $\mathfrak{L}$-acyclic chain functor $\mathbf{V}_{*}$ on $\mathfrak{L}$ and transformation of chain functors $\beta: \mathbf{V}_{*} \longrightarrow \mathbf{A}_{*} \mid \mathfrak{L}$ there exists a transformation of chain functors $\rho: \mathbf{V}_{*} \longrightarrow \mathbf{U}_{*}$ such that

$$
\alpha \rho=\beta .
$$

Regularity of $\mathbf{A}_{*}, \quad \mathbf{V}_{*}$ implies that of $\mathbf{U}_{*}$.

Proof: $\mathbf{U}_{*}$ as defined before is clearly $\mathfrak{L}$-acyclic (not just acyclic) by construction. Moreover $\alpha$ is a g-transformation. Since the assignment determined by $c \longmapsto \bar{c}$ in the proof of 4.3. is functorial and commutes with $l: V_{*}^{\prime}(X, A) \longrightarrow V_{*}(X, A), i^{\prime}$ : $V_{*}(A) \longrightarrow V_{*}^{\prime}(X, A)$ and the chain homotopies $D(H)$ of [5] D4), $\gamma$ turns out to be a transformation of chain functors, so that this part of 4.5. follows.-

The last assertion is immediate.-

Combining this with 4.1. allows us to talk about $\overline{\mathbf{U}}_{*}$ and we find a morphism

$$
\text { cone } \overline{\mathbf{U}}_{*} \longrightarrow \mathbf{A}_{*} \cup_{\zeta} \text { cone } \overline{\mathbf{U}}_{*}
$$

with $\zeta=\bar{\alpha}: \overline{\mathbf{U}}_{*} \longrightarrow \mathbf{A}_{*}$.

4.6. Lemma: There are g-transformations $\mathbf{U}_{*} \longrightarrow \mathbf{A}_{*}$, hence

$$
\text { cone } \mathbf{U}_{*} \longrightarrow \mathbf{A}_{*} \cup_{\zeta} \text { cone } \overline{\mathbf{U}}_{*} \text { and cone } \overline{\mathbf{U}}_{*} \longrightarrow \mathbf{A}_{*} \cup_{\zeta} \text { cone } \overline{\mathbf{U}}_{*} \text {. }
$$

One has

$$
\text { cone } \overline{\mathbf{U}}_{*}=\overline{\text { cone } \mathbf{U}_{*}} \text {. }
$$

Proof. The formation of the cone is functorial so that the assertion follows from the construction.-

On the subcategory $\mathfrak{L} \subset \mathfrak{K}^{2}$ we have the chain functor $\mathbf{U}_{*}\left(\mathbf{A}_{*} \cup_{\zeta}\right.$ cone $\left.\overline{\mathbf{U}}_{*}\right)$ which belongs to $\mathbf{A}_{*} \cup_{\zeta}$ cone $\overline{\mathbf{U}}_{*} \mid \mathfrak{L}$ in the sense of 4.3. (for each object in $\mathfrak{L}$ seperately). On the other hand we have cone $\mathbf{U}_{*} \mid \mathfrak{L}$ and claim: 
4.7. Lemma: cone $\overline{\mathbf{U}}_{*} \mid \mathfrak{L}$ satisfies the universality property of $\mathbf{U}_{*}\left(\mathbf{A}_{*} \cup_{\zeta}\right.$ cone $\left.\overline{\mathbf{U}}_{*}\right)$ in 4.5 .

Proof: The proof is accomplished in three steps:

1) Suppose $\mathbf{K}_{*}$ in theorem 4.1. is $\mathfrak{L}$ - acyclic, then $\overline{\mathbf{K}}_{*}$ is $\mathfrak{L}$-acyclic.

Proof: According to 4.1. $\tau: \mathbf{K}_{*} \subset \overline{\mathbf{K}}_{*} \mid \mathfrak{L}$ is a strong deformation retract. So 1$)$ follows.- 2) Let $A_{*} \cup_{\zeta}$ cone $\bar{U}_{*}(K, L) \quad(K, L) \in \mathfrak{L}$ be given, then there exists a retraction

$$
r: A_{*} \cup_{\zeta} \text { cone } \bar{U}_{*}(K, L) \longrightarrow A_{*}(K, L) .
$$

Proof: Since $\bar{U}_{*}(K, L)$ is acyclic, hence (as a free chain complex) contractible, we can deform cone $\bar{U}_{*}(K, L)$ onto $\bar{U}_{*}(K, L)$. This implies the assertion.-

$3)$ Let $(K, L) \in \mathfrak{L}$ be any object, $V_{*}$ an acyclic chain complex and

$$
\beta: V_{*} \longrightarrow\left(A_{*} \cup_{\zeta} \text { cone } \bar{U}_{*}\right)(K, L)
$$

a given chain mapping. We are going to detect a

$$
\rho: V_{*} \longrightarrow\left(\text { cone } \bar{U}_{*}\right)(K, L)
$$

such that $\eta \rho=\beta$, where $\eta$ : cone $\bar{U}_{*}(K, L) \longrightarrow\left(A_{*} \cup_{\zeta}\right.$ cone $\left.\bar{U}_{*}\right)(K, L)$ is induced by $\zeta$.

Proof: According to 2) we have a retraction $r: A_{*} \cup_{\zeta}$ cone $\bar{U}_{*} \longrightarrow A_{*}$ and therefore a $\rho^{\prime}: V_{*} \longrightarrow U_{*}(K, L)$ such that $r \beta=\alpha \rho^{\prime}$.

Suppose $\beta(v)=a+c, a \in \mathbf{A}_{*}, c \in$ cone $\overline{\mathbf{U}}_{*}$, then $\alpha \rho^{\prime}(v)=r \beta(v)=a$, so that $a \in i m \alpha \subset \mathbf{U}_{*} \subset \overline{\mathbf{U}}_{*} \subset$ cone $\overline{\mathbf{U}}_{*}$, hence $\beta(v) \in i m \eta$.

As a result we detect a $\rho: V_{*} \longrightarrow$ cone $\bar{U}_{*}$ such that $\eta \rho=\beta$ as required.

Hence cone $\bar{U}_{*}(K, L)$ satisfies the universality condition of 4.3 . and cone $\overline{\mathbf{U}}_{*} \mid \mathfrak{L}$ that of 4.5.-

\section{Localizations}

The kind of localization which we are now developing for chain functors has been originally discovered by A. K. Bousfield [6] for spectra as well as for mappings between spectra, and, more recently, extended for axiomatically defined stable homotopy categories by the authors of [10].

Let $\mathbf{A}_{*}$ be any (regular or irregular) chain functor on a category $\mathfrak{K}^{2}$ of pairs of topological spaces (or of Boardman spectra) and $\mathfrak{L} \subset \mathfrak{K}^{2}$ any subcategory. We are going to localize $\mathbf{A}_{*}$ at $\mathfrak{L}$. There are localizations in the world of chain functors with as well as in the world of chain functors without compact carriers. They can be treated by the same procedure. In the preceding section we mentioned two interesting categories $\mathfrak{L}$ :

1) $\mathfrak{L}$ consists of one single object, or of one single map.

2) Let $\Phi$ be a homology theory, defined on the category of all chain functors. Associate with $\Phi$ the category $\mathfrak{L}=\mathfrak{L}_{\Phi}=\left\{(K, L) \mid \forall \mathbf{A}_{*} ; \Phi\left(\mathbf{A}_{*}\right)=0 \Rightarrow\right.$ $\left.H_{*}\left(\mathbf{A}_{*}\right)(K, L)=0\right\}$.

5.1. Definition: 1) A morphism of chain functors (cf. [5] §4) $\eta: \mathbf{B}_{*} \longrightarrow \mathbf{C}_{*}$ is an $\mathfrak{L}$--isomorphism whenever $(\eta \mid \mathfrak{L})_{*}: H_{*}\left(\mathbf{B}_{*}\right)\left|\mathfrak{L} \stackrel{\approx}{\underset{\sim}{\longrightarrow}} H_{*}\left(\mathbf{C}_{*}\right)\right| \mathfrak{L}$ is an isomorphism. 
2) $\mathbf{C}_{*}$ is $\mathfrak{L}$-local whenever one has $\left[\mathbf{V}_{*}, \mathbf{C}_{*}\right]=0$ for any $\mathfrak{L}$-acyclic chain functor $\mathbf{V}_{*}$ (cf. definition 4.4.) ( $\left[\mathbf{V}_{*}, \mathbf{C}_{*}\right]$ denoting the set of all chain homotopy classes of

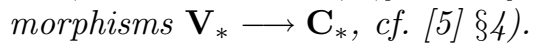

Alternatively $\mathbf{C}_{*}$ is $\mathfrak{L}$-local, whenever any $\mathfrak{L}$-isomorphism $f: \mathbf{V}_{*} \longrightarrow \tilde{\mathbf{V}}_{*}$ induces an isomorphism

$$
f^{*}:\left[\tilde{\mathbf{V}}_{*}, \mathbf{C}_{*}\right] \stackrel{\approx}{\underset{ }{\longrightarrow}}\left[\mathbf{V}_{*}, \mathbf{C}_{*}\right] .
$$

Observe that if the chain functor $\mathbf{B}_{*}$ is associated with a spectrum $\mathbf{B}$, then for any spectrum $\mathbf{E}$ one has

$$
\mathbf{B}_{*}(\mathbf{E})=H_{*}\left({ }^{\mathbf{E}} \mathbf{C}_{*}\right)(\mathbf{B}),
$$

so that the preceding definition agrees with the usual one ([1], [11], [13]).

According to 4.5 . we have an acyclic $\mathbf{U}_{*} \mid \mathfrak{L}=\mathbf{U}_{*}$ and a mapping $\alpha: \mathbf{U}_{*} \longrightarrow \mathbf{A}_{*} \mid \mathfrak{L}$. We set

$$
\mathfrak{L} \mathbf{A}_{*}=\overline{\mathbf{U}}_{*},
$$

where the $(\bar{\cdot})$-construction stems from 4.1 .

5.2. Lemma: $\mathbf{L}_{*}$ is $\mathfrak{L}$-acyclic. Regularity (resp. compact carriers) of $\mathbf{A}_{*}$ implies the same for ${ }_{\mathfrak{L}} \mathbf{A}_{*}$.

Proof: We have

$$
\mathfrak{L} A_{*}(L)=\bar{U}_{*}(L) \approx U_{*}(L),
$$

which is acyclic for any $L \in \mathfrak{L}$, so that $\overline{\mathbf{U}}_{*}$ is in view of 4.5. $\mathfrak{L}$-acyclic. The second assertion will be settled in $\S 6$ (theorem 6.8 . which will be independently verified). -

According to theorem 4.1. we have

$$
\zeta=\bar{\alpha}: \overline{\mathbf{U}}_{*} \longrightarrow \mathbf{A}_{*}
$$

and define

$$
\mathbf{A}_{\mathfrak{L} *}=\mathbf{A}_{*} \cup_{\zeta} \text { cone }\left(\mathfrak{L} \mathbf{A}_{*}\right)
$$

denoting by

$$
\eta: \mathbf{A}_{*} \longrightarrow \mathbf{A}_{\mathfrak{L} *}
$$

the mapping induced by $\zeta$.

As in the preceding section, the formation of the cone over a chain functor as well as that of the amalgamated sum carries immediately over from chain complexes ([8]) to chain functors.

5.3. Lemma: Let $\mathbf{V}_{*}$ be $\mathfrak{L}$-acyclic, then

$$
\left[\mathbf{V}_{*}, \mathbf{A}_{\mathfrak{L} *}\right]=0
$$

hence $\mathbf{A}_{\mathfrak{L} *}$ is $\mathfrak{L}$ - local.

Proof. Let $f: \mathbf{V}_{*} \longrightarrow \mathbf{A}_{*} \cup_{\zeta}$ cone $\overline{\mathbf{U}}_{*}$ be any mapping of chain functors, $\mathbf{V}_{*} \mathfrak{L}$ -acyclic. According to [5] 4.5. we can assume that $f$ is a g-transformation. Due to 4.5. we find a factorization 


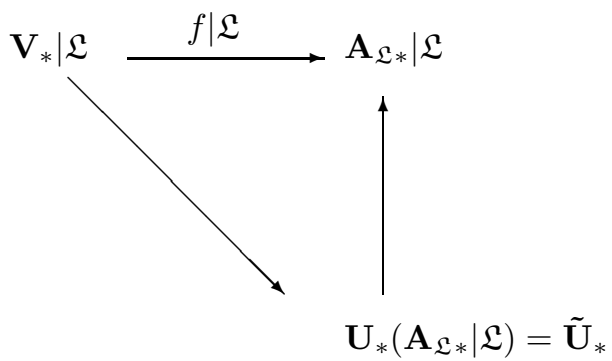

hence according to theorem 4.1. a factorization

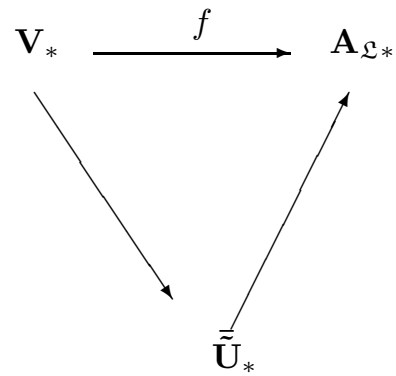

However according to 4.7 . we are allowed to use cone $\mathbf{U}_{*}$ instead of $\tilde{\mathbf{U}}_{*}$ and therefore, because of 4.6 ,

$$
\overline{\tilde{\mathbf{U}}}_{*}=\operatorname{cone} \overline{\mathbf{U}}_{*}
$$

with

$$
\mathbf{U}_{*}=\mathbf{U}_{*}\left(\mathbf{A}_{*}\right) \mid \mathfrak{L} .
$$

So we have a factorization

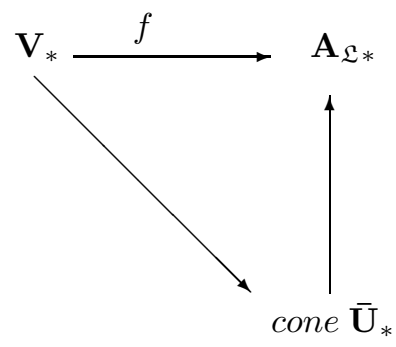

implying that $f \simeq 0$, since the cone over everything is always acyclic.- 
5.4. Lemma: $\eta$ is an $\mathfrak{L}$-isomorphism.

Proof: 1) To any cycle $z \in A_{\mathfrak{L} *}(L), L \in \mathfrak{L}$ there exists a $z^{\prime} \sim z, \quad z^{\prime} \in i m \eta$, hence $H_{*}(\eta(L))$ is epic.

Proof. Suppose $v \in$ cone $U_{*}(L), \quad d v \in \zeta\left(U_{*}\right)$, then, since $\mathbf{U}_{*}$ is acyclic, $d v$ is bounding in $U_{*}(L), \zeta^{-1}(d v)=d u$, so that $d v=d \zeta(u)$. If $z=u_{A}+v, u_{A} \in i m \eta, v$ as before, then we have

$$
z=u_{A}+(v-u)+u .
$$

Since $v-u \sim 0$ (as a cycle in cone $U_{*}$ ) the assertion follows.

2) Let $z \in A_{*}(L), \quad L \in \mathfrak{L}$ be a cycle, such that $z$ bounds, then the same argument ensures that $z$ bounds already in $A_{*}(L)$. Hence $H_{*}(\eta(L))$ is monic.-

So $H_{*}(\eta(L))$ is an isomorphism.-

We summarize:

5.5. Theorem: Let $\mathbf{A}_{*}$ be any chain functor, $\mathfrak{L} \subset \mathfrak{K}^{2}$ any subcategory, then there exists a (with respect to $\mathbf{A}_{*}$ ) natural exact sequence of chain functors

$$
{ }_{\mathfrak{L}} \mathbf{A}_{*} \stackrel{\zeta}{\longrightarrow} \mathbf{A}_{*} \stackrel{\eta}{\longrightarrow} \mathbf{A}_{\mathcal{L} *}
$$

where $\mathfrak{L}_{*} \mathbf{A}_{*}$ is $\mathfrak{L}$-acyclic, $\mathbf{A}_{\mathfrak{L} *} \mathfrak{L}$-local and $\eta$ an $\mathfrak{L}$-isomorphism.

$\mathbf{A}_{\mathfrak{L}}, \quad \mathfrak{L} \mathbf{A}_{*}$ are regular (resp. have compact carriers), whenever we start with a regular $\mathbf{A}_{*}$ (resp. with one having compact carriers).

Proof: The existence of (4) is a consequence of the preceding lemmas, while the exactness (to be understood in an obvious sense) follows from (1)-(3). The second assertion will be treated by theorem 6.8. (cf. 5.2.) -

5.6. Corollary: Let $\mathbf{A}_{*}$ be as in 5.5., $E \in \mathfrak{K}$ any object, then there exists an exact sequence

$$
{ }_{E} \mathbf{A}_{*} \stackrel{\zeta}{\longrightarrow} \mathbf{A}_{*} \stackrel{\eta}{\longrightarrow} \mathbf{A}_{E *}
$$

with $E$-acyclic ${ }_{E} \mathbf{A}_{*}$ and $E$-local $\mathbf{A}_{E *}$.

In [5] we mentioned the possibility to extend the definition of a chain functor to a category of spectra (i.e. to the Boardman category $\mathfrak{B}$ resp. to the category $\mathfrak{B}^{2}$ of pairs of spectra). We can immediately rephrase 5.6. for this case, accomplishing

5.7. Corollary: Let $\mathbf{A}_{*}$ be a chain functor on $\mathfrak{B}^{2}, E \in \mathfrak{B}$, then there exists an exact sequence (5).

This covers the localization process described in [6] by A. Bousfield.

We return to example 2) at the beginning of this section:

If the category on which our chain functors are defined is the Boardman category $\mathfrak{B}$, any homology theory $\Phi$ which is defined on $\mathfrak{B}$ admits an extension over the category of all chain functors by setting $\Phi\left(\mathbf{A}_{*}\right)=\Phi\left(\left|\mathbf{A}_{*}\right|\right)$, where $\left|\mathbf{A}_{*}\right|$ is the realization of the chain functor in the sense of theorem 1.1. in [3]. Moreover $\Phi$ has a classifying spectrum $\mathbf{E}$ so that $\mathbf{E}_{*}=\Phi$. Now it turns out easily that $\mathfrak{L}_{\Phi}$-localization is the same as $\mathbf{E}$-localization. 
Suppose now that $\Phi$ is a homology theory on $\mathfrak{K}^{2} \subset \mathbf{T o p}^{2}$, then we define $\mathfrak{L}_{\Phi}=$ $\left\{(K, L) \mid \forall X \in \mathfrak{K} ; \Phi(X)=0 \Rightarrow \mathbf{X}_{*}(K, L)=0\right\}$, where $\mathbf{X}_{*}$ denotes the homology theory associated with $X$.

This provides us with a second access to the localization of a chain functor $\mathbf{A}_{*}$ defined on a category $\mathfrak{K}^{2}\left(\subset\right.$ Top $\left.^{2}\right)$ with respect to a spectrum $\mathbf{E} \in \mathfrak{B}$ (not being an object of $\mathfrak{K}^{2}$ !) by using the homology theory $\mathbf{E}_{*}=\Phi$ and localizing $\mathbf{A}_{*}$ with respect to $\mathfrak{L}_{\Phi}$ in the sense of 5.5 .

As a result we get:

5.8. Corollary: Let $\mathbf{A}_{*}$ be as in 5.5., $\mathbf{E} \in \mathfrak{B}$ a spectrum, then there exists an exact localization sequence (5).

\section{Localizations and tensor products}

In $\S 3$ we introduced a chain functor $\mathbb{Z}_{*}$ to which the localization process can be applied. Hence for given subcategory $\mathfrak{L} \subset \mathfrak{K}^{2}$ we obtain $\mathbb{Z}_{*}$ and $\mathbb{Z}_{\mathfrak{L} *}$. It turns out that for any chain functor $\mathbf{A}_{*}$ the exact sequence (4) in $\S 5$ originates from the sequence

$$
\mathfrak{L} \mathbb{Z}_{*} \stackrel{\zeta}{\longrightarrow} \mathbb{Z}_{*} \stackrel{\eta}{\longrightarrow} \mathbb{Z}_{\mathfrak{L} *}
$$

by tensoring with $\mathbf{A}_{*}$.

Recall that the chain functor $\mathbf{U}_{*}$ of 4.5 . is isomorphic to $\mathbf{A}_{*} \mid \mathfrak{L} \otimes D_{*}$ (cf. proof of 4.3.).

6.1. Lemma: For any chain functor $\mathbf{A}_{*}$ we have a natural homotopy equivalence

$$
\mathbf{A}_{*} \otimes \mathfrak{L} \mathbb{Z}_{*} \stackrel{\simeq}{\mathfrak{L}} \mathbf{A}_{*} .
$$

Proof: At first we introduce and recall some notations which are mostly taken from the proof of 4.1.:

There are elements $\left\{z_{X} ; z_{K} \otimes x\right\},\left\{0 ; z_{K} \otimes d x\right\}$ in $\mathfrak{L} \mathbb{Z}_{n}(X, A), n=0,-1$ as well as $\mathbf{a}=\left\{a ; f_{\#}(a) \otimes x\right\}, \mathbf{a}=\left\{a ; a_{f} \otimes d x\right\}, \quad f_{\#}(a)=0$ for all $f:(X, A) \longrightarrow(E, F) \in \mathfrak{L}$ , which are continuous mappings or words in the sense of the proof of 4.1. (II), $\mathbf{a} \in \mathfrak{L} A_{n}(X, A)$.

Moreover in cone $K_{*}^{0}(X, A) \subset \mathfrak{L} A_{*}(X, A)$ we have elements $\Delta\left(\left\{0 ; a_{f} \otimes d x\right\}\right)$, satisfying $d \Delta\left(\left\{0 ; a_{f} \otimes d x\right\}\right)=\left\{0 ; a_{f} \otimes d x\right\}-\Delta\left(\left\{0 ; d a_{f} \otimes d x\right\}\right)$. In $\mathfrak{L} \mathbb{Z}_{*}$ we encounter chains $\Delta\left(\left\{0 ; z_{K} \otimes d x\right\}\right)=\Delta \in K_{*}^{0}(X, A)$ and $\left\{z_{X} ; z_{K} \otimes x\right\}$ with the same boundary. We denote the cycle $\left\{z_{X} ; z_{K} \otimes x\right\}-\Delta$ by $\nabla \in \mathfrak{L} \mathbb{Z}_{0}(X, A)$.

Using these notations we define

$$
\eta: A_{*} \otimes \mathfrak{L} \mathbb{Z}_{*} \longrightarrow \mathfrak{L} \mathbf{A}_{*}
$$

by setting

$$
\begin{gathered}
\eta\left(a \otimes\left\{z_{X} ; z_{K} \otimes x\right\}\right)=\left\{a ; f_{\#}(a) \otimes x\right\} \\
\left.\eta\left(a \otimes\left\{0 ; z_{K} \otimes d x\right\}\right)=\left\{0 ; f_{\#}(a) \otimes d x\right\}\right) \\
\eta(a \otimes \Delta)=\Delta\left\{0 ; f_{\#}(a) \otimes d x\right\}
\end{gathered}
$$


One checks immediately that $\eta$ is a natural chain mapping. The inverse

$$
\zeta: \mathfrak{L} \mathbf{A}_{*} \longrightarrow \mathbf{A}_{*} \otimes \mathfrak{L} \mathbb{Z}_{*}
$$

is defined by setting

$$
\zeta\left(\left\{a ; f_{\#}(a) \otimes x\right\}\right)=a \otimes \nabla
$$

while on all elements in cone $K_{*}^{0}$ we set $\zeta=0$.

This establishes again a natural chain mapping.

We have

$$
\begin{aligned}
& \zeta \eta\left(a \otimes\left\{z_{X} ; z_{K} \otimes x\right\}\right)=a \otimes \nabla=a \otimes\left\{z_{X} ; z_{K} \otimes x\right\}-a \otimes \Delta, \\
& \eta \zeta\left(\left\{a ; f_{\#}(a) \otimes x\right\}\right)=\left\{a ; f_{\#}(a) \otimes x\right\}-\Delta\left(\left\{0 ; f_{\#}(a) \otimes d x\right\}\right) .
\end{aligned}
$$

Here, as in all remaining cases, we observe that

$$
\zeta \eta()-()
$$

resp.

$$
\eta \zeta()-()
$$

are elements of $A_{*} \otimes$ cone $K_{*}^{0}\left(K_{*}^{0}\right.$ understood for $\mathfrak{L} \mathbb{Z}_{*}$ ) resp. of cone $K_{*}^{0}$ (now for $A_{*}$ ). Since cone $K_{*}^{0}$ (contained in $\mathfrak{s} A_{*}$ ) as well as $A_{*} \otimes$ cone $K_{*}^{0}$ are acyclic (the latter e.g. due to the Künneth-formula), we conclude that

$$
\begin{gathered}
\zeta \eta \simeq 1 \\
\eta \zeta \simeq 1 .
\end{gathered}
$$

This chain homotopy can of course be realized in detail, which we will accomplish for $\zeta \eta\left(a \otimes\left\{z_{X} ; z_{K} \otimes x\right\}\right)$, The remaining cases are settled by a similar procedure. We need a $\Gamma(u)$ for $u=a \otimes\left\{z_{X} ; z_{K} \otimes x\right\}$ such that $\zeta \eta(u)-u=d \Gamma(u)+\Gamma d u$. Observe that $\zeta \eta(d u)=d a \otimes\left\{z ; z_{K} \otimes x\right\}-(-1)^{n-1} d a \otimes\left\{0 ; z_{K} \otimes d x\right\}+(-1)^{n-1} d a \otimes$ $\left\{0 ; z_{K} \otimes d x\right\}-d a \otimes \Delta$. Since $-(-1)^{n-1} d a \otimes\left\{0 ; z_{K} \otimes d x\right\}-d a \otimes \Delta=w$ is a cycle in $A_{*} \otimes$ cone $K_{*}^{0}$, hence bounding, there exists a $\Gamma(d u)$ in $A_{*} \otimes$ cone $K_{*}^{0}$, such that

$$
w=d \Gamma(d u)=\zeta \eta(d u)-d u
$$

Now we have

$$
\zeta \eta(u)-u-\Gamma(d u)=-a \otimes \Delta-\Gamma(d u)=w^{\prime}
$$

with $w^{\prime} \in A \otimes$ cone $K_{*}^{0}$. Since $w^{\prime}$ is a cycle, there exists a $d \Gamma(u)$ satisfying $d \Gamma(u)=$ $w^{\prime}=\zeta \eta(u)-u-\Gamma(d u)$.

This can be performed in a natural way, establishing a $\Gamma(u)$ such that

$$
d \Gamma(u)+\Gamma(d u)=\zeta \eta(u)-u .
$$

Up to this point $\eta$ as well as $\zeta$ are mappings of chain complexes (rather than of chain functors). However endowing them with the structure of chain functor 
transformations is immediate.

This completes the proof of 6.1 .-

6.2. Lemma: For any chain functor $\mathbf{A}_{*}$ we have a natural isomorphism

$$
\mathbf{A}_{*} \otimes \text { cone }_{\mathfrak{L}} \mathbb{Z}_{*} \approx \text { cone }\left(\mathbf{A}_{*} \otimes \mathfrak{L} \mathbb{Z}_{*}\right)
$$

Proof: We have for any chain functor $\mathbf{C}_{*}$ an isomorphism

$$
\left(\text { cone } C_{*}\right)(X) \approx C_{*}(X) \otimes B_{*}
$$

where $B_{*}=\sum D_{*}$ (denoting by $\sum$ the suspension), i.e. $B_{1}=\langle x\rangle, B_{0}=\langle d x\rangle, B_{n}=$ 0 elsewhere $(\S 4)$. Hence we detect a sequence of isomorphisms

$$
\begin{aligned}
& \mathbf{A}_{*} \otimes \text { cone }_{\mathfrak{L}} \mathbb{Z}_{*} \approx \mathbf{A}_{*} \otimes\left(\mathfrak{L} \mathbb{Z}_{*} \otimes B_{*}\right) \approx \\
& \approx\left(\mathbf{A}_{*} \otimes \mathfrak{L} \mathbb{Z}_{*}\right) \otimes B_{*} \approx \text { cone }\left(\mathbf{A}_{*} \otimes \mathfrak{L} \mathbb{Z}_{*}\right) .-
\end{aligned}
$$

6.3. Lemma We have a natural homotopy equivalence

$$
\mathbf{A}_{\mathfrak{L} *} \simeq \mathbf{A}_{*} \otimes \mathbb{Z}_{\mathfrak{L} *}
$$

Proof: We have

$$
\mathbf{A}_{\mathfrak{L} *} \approx \mathbf{A}_{*} \cup_{\zeta} \text { cone }\left(\mathfrak{L} \mathbf{A}_{*}\right) \simeq
$$

$$
\mathbf{A}_{*} \cup_{\zeta} \text { cone }\left(\mathbf{A}_{*} \otimes \mathfrak{L} \mathbb{Z}_{*}\right) \approx
$$

$$
\begin{gathered}
\mathbf{A}_{*} \cup_{\zeta}\left(\mathbf{A}_{*} \otimes \text { cone }_{\mathfrak{L}} \mathbb{Z}_{*}\right) \approx \mathbf{A}_{*} \otimes\left(\mathbb{Z}_{*} \cup_{\zeta} \text { cone }\left(\mathfrak{s} \mathbb{Z}_{*}\right)\right) \approx \\
\approx \mathbf{A}_{*} \otimes \mathbb{Z}_{\mathfrak{L}_{*} \cdot-}
\end{gathered}
$$

6.4. Lemma: $1_{\mathbf{A}_{*}} \otimes \eta: \mathbf{A}_{*} \otimes \mathbb{Z}_{*} \longrightarrow \mathbf{A}_{*} \otimes \mathbb{Z}_{\mathfrak{L}_{*}}$ is an $\mathfrak{L}$ - isomorphism. Proof: Follows immediately.-

We summarize this in the following main theorem:

6.5. Theorem: Let $\mathbf{A}_{*}$ be any chain functor, $\mathfrak{L} \subset \mathfrak{K}^{2}$ any subcategory, then the exact $\mathfrak{L}$-localization sequence of $\mathbf{A}_{*}$ is given by:
$\mathbf{A}_{*} \otimes \mathfrak{L} \mathbb{Z}_{*}$
$1 \otimes \zeta$
$\mathbf{A}_{*} \otimes \mathbb{Z}_{*}$
$1 \otimes \eta$
$\mathbf{A}_{*} \otimes \mathbb{Z}_{\mathfrak{L} *}$
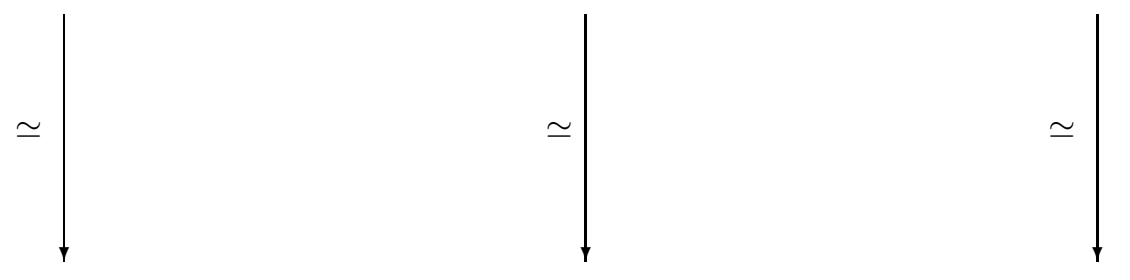
$\mathfrak{s} \mathbf{A}_{*}$
$\mathbf{A}_{*}$
$\mathbf{A}_{\mathfrak{L} *}$ 
Proof: The only remaining fact which needs proof is that $1 \otimes \zeta$ resp. $1 \otimes \eta$ are in fact the mappings $\zeta$ resp. $\eta$ of $\S 5(2)$ for $\mathbf{A}_{*}$, but this is due to the construction straightforward.-

6.6. Corollary: Let $E \in \mathfrak{K}$ be any object, then the exact $E$-localization sequence of a chain functor takes the form

$$
\mathbf{A}_{*} \otimes{ }_{E} \mathbb{Z}_{*} \longrightarrow \mathbf{A}_{*} \longrightarrow \mathbf{A}_{*} \otimes \mathbb{Z}_{E *} .
$$

6.7. Corollary: There exist natural homotopy equivalences

$$
\mathbf{A}_{\mathfrak{L} *} \otimes \mathbf{B}_{\mathfrak{L} *} \simeq\left(\mathbf{A}_{*} \otimes \mathbf{B}_{*}\right)_{\mathfrak{L} *} \simeq \mathbf{A}_{*} \otimes \mathbf{B}_{\mathfrak{L} *} .
$$

Proof: One has:

$$
\left(\mathbf{A}_{*} \otimes \mathbf{B}\right)_{\mathfrak{L} *} \simeq\left(\mathbf{A}_{*} \otimes \mathbf{B}_{*}\right) \otimes \mathbb{Z}_{\mathfrak{L} *} \simeq \mathbf{A}_{*} \otimes\left(\mathbf{B}_{*} \otimes \mathbb{Z}_{\mathfrak{L}_{*}}\right) .
$$

However since $\left(\mathbf{A}_{\mathfrak{L} *}\right)_{\mathfrak{L} *} \simeq \mathbf{A}_{\mathfrak{L} *}$ as an immediate consequence of the definition of an $\mathfrak{L}$-local object in definition 5.1., in particular $\mathbb{Z}_{\mathfrak{L} *} \otimes \mathbb{Z}_{\mathfrak{L} *} \simeq\left(\mathbb{Z}_{\mathfrak{L} *}\right)_{\mathfrak{L} *}$, we have

$$
\begin{aligned}
& \mathbf{A}_{*} \otimes \mathbf{B}_{*} \otimes \mathbb{Z}_{\mathfrak{L} *} \simeq \mathbf{A}_{*} \otimes \mathbf{B}_{*} \otimes \mathbb{Z}_{\mathfrak{L} *} \otimes \mathbb{Z}_{\mathfrak{L} *} \\
\simeq & \left(\mathbf{A}_{*} \otimes \mathbb{Z}_{\mathfrak{L} *}\right) \otimes\left(\mathbf{B}_{*} \otimes \mathbb{Z}_{\mathfrak{L} *}\right) \simeq \mathbf{A}_{\mathfrak{L} *} \otimes \mathbf{B}_{\mathfrak{L} *} .
\end{aligned}
$$

Remarks: 1) Since there is no spectrum $\left|\mathbb{Z}_{*}\right|$ associated with the irregular-chainfunctor $\mathbb{Z}_{*}$, theorem 6.5. has no immediate counterpart for spectra, unless one agrees to define tensor products $\left|\mathbf{B} \otimes \mathbf{C}_{*}\right|$ between spectra $\mathbf{B}$ and chain functors $\mathbf{C}_{*}$, which is of course possible.-

2) Corollary 6.7. ensures that, unlike the $\wedge$-product, the $\otimes$ - product turns out to be compatible with localizations.

6.8. Theorem If $\mathbf{A}_{*}$ is regular (resp. has compact carriers), then ${ }_{\mathfrak{L}} \mathbf{A}_{*}$ and $\mathbf{A}_{\mathfrak{L} *}$ are regular chain functors (resp. they have compact carriers), i.e. the derived homology is a homology theory (with, resp. without compact carriers).

Proof: If the chain functors $\mathbf{K}_{*}$ and $\mathbf{C}_{*}$ in 4.1. are regular, we have that $\overline{\mathbf{K}}_{*}$ satisfies the requirement that $l, i^{\prime}$ and $\left((X, A) \subset(Y, B)_{\#}\right)$ are inclusions onto direct summands (cf. 4.1. 3)). Hence $\mathfrak{i} \mathbf{A}_{*}$ has this property.

Moreover it is immediate that this property carries over to $\mathbf{A}_{\mathfrak{L}_{*}}(\S 5$ (2)).

So it remains to deal with excision. To this end we retain the notation of the proof of 6.1. and consider $\mathbf{A}_{*} \otimes_{\mathfrak{L}} \mathbb{Z}_{*}$. Suppose that $h:(X, A) \longrightarrow(X / A, \star)$ is an excision map, then the second component in the mapping of tensor products

$$
h_{\#}:\left(A_{*} \otimes \mathfrak{L} \mathbb{Z}_{*}\right)(X, A) \longrightarrow\left(A_{*} \otimes \mathfrak{L} \mathbb{Z}_{*}\right)(X / A, \star)
$$

does not change: Due to the definition of tensorproducts in $\S 1(1)$ as well as that of $\mathbb{Z}_{*}$ in $\S 3$, only $h: X \longrightarrow X / A$ enters, inducing an isomorphism even on the chain level. So one has to deal merely with $h_{\#}$ on $\mathbf{A}_{*}$, which, by assumption induces an isomorphism of the derived homology. Consequently $h_{\#}$ on $\mathbf{A}_{*} \otimes_{\mathfrak{L}} \mathbb{Z}_{*}$ induces an isomorphism of the derived homology of $\mathbf{A}_{*} \otimes \mathfrak{z} \mathbb{Z}_{*}$ and therefore, because of 6.1., of ${ }_{\mathfrak{L}} \mathbf{A}_{*}$. By a similar argument we ensure, employing 6.3., that $\mathbf{A}_{\mathfrak{L} *}$ satisfies the same excision property as $\mathbf{A}_{*}$. 
According to 4.1. $\mathfrak{L} \mathbf{A}_{*}=\overline{\mathbf{U}}_{*}$ can be assumed to have compact carriers; the same holds for cone $\overline{\mathbf{U}}_{*}$ and consequently $\mathbf{A}_{*} \cup_{\zeta}$ cone $\overline{\mathbf{U}}_{*}$ inherit this property from that of $\mathbf{A}_{*}$ and of cone $\overline{\mathbf{U}}_{*}$.-

Although tensor products do not appear explicitely in the formulation of 6.8., it nevertheless displays itself as an implication of 6.1., 6.3.. We can apply 6.8. to regain the existence of Bousfield-localizations of spectra (rather than of chain functors):

6.9. Corollary: Let $\mathfrak{L} \subset \mathfrak{K}^{2}, \mathfrak{K}=\mathfrak{B}$ be as before, $\mathbf{A}$ a spectrum, then there exists an exact sequence of spectra

$$
\mathfrak{L} \mathbf{A} \stackrel{\zeta}{\longrightarrow} \mathbf{A} \stackrel{\eta}{\longrightarrow} \mathbf{A}_{\mathfrak{L}}
$$

with $\mathfrak{L}$ - acyclic $\mathfrak{L} \mathbf{A}, \mathfrak{L}$ - local $\mathbf{A}_{\mathfrak{L}}$ and $\mathfrak{L}$-isomosphic $\eta$. Proof: The spectrum $\mathbf{A}$ is associated with a regular chain functor $\mathbf{C}_{*}={ }^{\mathbf{A}} \mathbf{C}_{*}$. Theorem 6.8. in combination with [3] theorem 1.1. ensure that $\left|{ }_{\mathfrak{L}} \mathbf{C}_{*}\right|=\mathfrak{L} \mathbf{A}$ as well as $\left|\mathbf{C}_{\mathfrak{L} *}\right|$ exists. The remaining points are settled by standard arguments.-

6.9. Corollary: (A. Bousfield [6]) Let $\mathbf{A}, \mathbf{B} \in \mathfrak{B}$ be spectra, then there exists a localization sequence (2), with $\mathbf{E}$ replacing $\mathfrak{L}$.

We are presently not treating the unstable localization theory of [7].

\section{References}

[1] F. Adams, "Stable homotopy and generalized homology". Univ. of Chicago Press, Chicago 1974

[2] F. W. Bauer, Generalized homology theories and chain functors. Annali di Matematica pura ed appl.(IV) Vol.CLV (1989) 143-191

[3] _- - Classifying spectra for generalized homology theories. Annali di Matematica pura ed appl. (IV) Vol. CLXIV (1993) 365-399

[4] - - , The Boardman category of spectra, chain complexes and (co-)localizations. Homology, Homotopy and Applications Vol. 1 No. 3 (1999) $95-116$

[5] _- - Chain functors with isomorphic homology

[6] A.K. Bousfield, Localization of a spectrum with respect to homology. Topology 18 (1979) 257-281

[7] —" - Homotopical localizations of spaces. Am. Journ. of Math. 119 (1997) $1321-1354$

[8] A. Dold, "Lectures on Algebraic Topology". Springer, Berlin-HeidelbergNewYork (1972) 
[9] A. D. Elmendorf, I. Kriz, M. A. Mandell, J. P. May " Rings, modules and algebras in stable homotopy theory". Amer. Math. Soc. Surveys and Monographs Vol. 47 (1996)

[10] M. Hovey, J. H. Palmieri, N. P. Strickland Axiomatic stable homotopy theory. Vol. 128, Mem. Am. Math. Soc. no. 610 AMS (1997)

[11] H. R. Margolis, "Spectra and the Steenrod Algebra". North- Holland Mathem. Library, Amsterdam-NewYork-Oxford (1983)

[12] D. C. Ravenel, Nilpotence and periodicity in stable homotopy theory. Ann. Math. Stud. no. 128, Princeton Univ. Press, Princeton NJ, (1992)

[13] R. Switzer, "Algebraic Topology-Homotopy and Homology". Springer, BerlinHeidelberg-NewYork (1975)

This article may be accessed via WWW at http://www.rmi.acnet.ge/hha/ or by anonymous ftp at

ftp://ftp.rmi.acnet.ge/pub/hha/volumes/2001/n3/n3.(dvi,ps,dvi.gz,ps.gz)

Friedrich W. Bauer f.w.bauer@mathematik.uni-frankfurt.de

Johann-Wolfgang Goethe Universität

Fachbereich Mathematik

Robert-Mayer Strasse 8-10

60054 Frankfurt a. M.

Germany 